\title{
Tuning and auto-tuning of fractional order controllers for industry applications
}

\author{
Concepción A. Monje $^{\mathrm{a}, *}$, Blas M. Vinagre ${ }^{\mathrm{b}}$, Vicente Feliu' ${ }^{\mathrm{c}}$, YangQuan Chen ${ }^{\mathrm{d}}$ \\ ${ }^{a}$ Department of Systems Engineering and Automatics, University Carlos III of Madrid, Av. Universidad 30, 28911 Leganés, Madrid, Spain \\ ${ }^{\mathrm{b}}$ Department of Electronics and Electromechanical Engineering, Industrial Engineering School, University of Extremadura, \\ Av. Elvas s/n, 06071 Badajoz, Spain \\ ${ }^{\mathrm{c}}$ Department of Electrical, Electronic and Automatic Engineering, Higher Technical School of Industrial Engineering, University of Castilla-La Mancha, \\ Av. Camilo José Cela s/n, 13071 Ciudad Real, Spain \\ ${ }^{\mathrm{d}}$ Center for Self-Organizing and Intelligent System, Department of Electrical and Computer Engineering, Utah State University, UMC 4160, \\ College of Engineering, 4160 Old Main Hill, Logan, UT 84322-4160, USA
}

Received 10 November 2006; accepted 22 August 2007

Available online 22 October 2007

\begin{abstract}
This paper deals with the design of fractional order $P I^{\lambda} D^{\mu}$ controllers, in which the orders of the integral and derivative parts, $\lambda$ and $\mu$, respectively, are fractional. The purpose is to take advantage of the introduction of these two parameters and fulfill additional specifications of design, ensuring a robust performance of the controlled system with respect to gain variations and noise. A method for tuning the $P I^{\lambda} D^{\mu}$ controller is proposed in this paper to fulfill five different design specifications. Experimental results show that the requirements are totally met for the platform to be controlled. Besides, this paper proposes an auto-tuning method for this kind of controller. Specifications of gain crossover frequency and phase margin are fulfilled, together with the iso-damping property of the time response of the system. Experimental results are given to illustrate the effectiveness of this method.
\end{abstract}

(C) 2007 Elsevier Ltd. All rights reserved.

Keywords: Auto-tuning; PID controller; Fractional order controller; Gain variations; Robust control

\section{Introduction}

Nowadays, the better understanding of the potential of fractional calculus and the increasing number of studies related to the applications of fractional order controllers in many areas of science and engineering have led to the importance of studying aspects such as the analysis, design, tuning and implementation of these controllers.

Fractional calculus is a generalization of the integration and differentiation to the non-integer (fractional) order fundamental operator ${ }_{a} \mathrm{D}_{t}^{\alpha}$, where $a$ and $t$ are the limits and $\alpha(\alpha \in \mathbb{R})$ is the order of the operation. Among many different definitions, two commonly used for the general fractional integro-differential operation are the Grünwald-Letnikov (GL) definition and the Riemann-Liouville

\footnotetext{
*Corresponding author. Tel.: + 34916248813 ; fax: + 34916249430 .

E-mail address: cmonje $@$ ing.uc3m.es (C.A. Monje).
}

(RL) definition (Podlubny, 1999a). The GL definition is

${ }_{a} \mathrm{D}_{t}^{\alpha} f(t)=\lim _{h \rightarrow 0} h^{-\alpha} \sum_{j=0}^{[(t-a) / h]}(-1)^{j}\left(\begin{array}{l}\alpha \\ j\end{array}\right) f(t-j h)$,

where $[\cdot]$ means the integer part, while the RL definition is ${ }_{a} \mathrm{D}_{t}^{\alpha} f(t)=\frac{1}{\Gamma(n-\alpha)} \frac{\mathrm{d}^{n}}{\mathrm{~d} t^{n}} \int_{a}^{t} \frac{f(\tau)}{(t-\tau)^{\alpha-n+1}} \mathrm{~d} \tau$

for $(n-1<\alpha<n)$ and where $\Gamma(\cdot)$ is Euler's gamma function.

For convenience, Laplace domain notion is commonly used to describe the fractional integro-differential operation. The Laplace transform of the RL fractional derivative/integral (2) under zero initial conditions for order $\alpha(0<\alpha<1)$ is given by

$£\left\{{ }_{a} \mathrm{D}_{t}^{ \pm \alpha} f(t)\right\}=s^{ \pm \alpha} F(s)$.

In theory, control systems can include both the fractional order dynamic system to be controlled and the 
fractional order controller. However, in control practice, more common is to consider the fractional order controller. This is due to the fact that the plant model may have been already obtained as an integer order model in the classical sense.

In this line, the objective of this work is to apply fractional order control (FOC) for industrial applications, introducing a fractional order controller to improve the system control performance and taking the most of the fractional orders of the controller.

It is important to realize that there is a very wide range of control problems and consequently also a need for a wide range of design techniques. There are already many tuning methods available but a replacement of the Ziegler-Nichols method is long overdue. On the research side it appears that the development of design methods for integer order control, and specially Proportional-Integral-Derivative (PID) control, is approaching the point of diminishing returns. There are some difficult problems that remain to be solved.

Therefore, this paper proposes the application of fractional calculus as an alternative option to solve some of the control problems that can arise when dealing with industrial applications, as will be commented later. On the one hand, a new method for the design of fractional order controllers is proposed, and more concretely for the tuning of a generalized $P I^{\lambda} D^{\mu}$ controller of the form:

$C(s)=k_{p}+\frac{k_{i}}{s^{\lambda}}+k_{d} s^{\mu}$,

where $\lambda$ and $\mu$ are the fractional orders of the integral and derivative parts of the controller, respectively. Since this kind of controller has five parameters to tune $\left(k_{p}, k_{d}, k_{i}, \lambda, \mu\right)$, up to five design specifications for the controlled system can be met, that is, two more than in the case of a conventional PID controller, where $\lambda=1$ and $\mu=1$. It is essential to study which specifications are more interesting as far as performance and robustness are concerned, since it is the aim to obtain a controlled system robust to uncertainties of the plant model, load disturbances and high frequency noise. All these constraints will be taken into account in the tuning technique of the controller in order to take advantage of the introduction of the fractional orders.

On the other hand, another approach of this work refers to the auto-tuning of fractional order controllers. As commented before, nowadays many research efforts related to the applications of fractional order controllers have concentrated on various aspects of control analysis and synthesis. However, in practical industrial settings, a similar auto-tuning procedure for this kind of controller is rarely found but in strong demand. Therefore, the ultimate goal is to develop a method to auto-tune a generalized $P I^{\lambda} D^{\mu}$ controller that allows the fulfillment of robustness constraints and whose implementation process is simple and reliable.
The implementation and application of these fractional order controllers for industrial purposes are other remarkable aspects aimed in this work, showing the results obtained when testing the controller in different experimental platforms.

This paper is organized as follows. First, Section 2 shortly reviews the state of the art of FOC and introduces some considerations on the implementation of fractional order controllers. The tuning method proposed for fractional order $P I^{\lambda} D^{\mu}$ controllers is described in Section 3, showing the results obtained when controlling an experimental platform with the controller designed. Section 4 presents an auto-tuning method for this kind of controller, whose experimental results are also shown in the section. Finally, some relevant concluding remarks are presented in Section 5.

\section{Fractional order control}

\subsection{A review}

Even though the idea of fractional order operators is as old as the idea of integer order ones, it has been in the last decades when the use of fractional order operators and operations has become more and more popular among many research areas. The theoretical and practical interest of these operators is nowadays well established, and its applicability to science and engineering can be considered as an emerging new topic. Even if they can be thought of as somehow ideal, they are, in fact, useful tools for both the description of a more complex reality and the enlargement of the practical applicability of the common integer order operators. Among these fractional order operators and operations, the fractional integro-differential operators (fractional calculus) are specially interesting in automatic control and robotics, among others, as detailed next.

Maybe the first mention of the interest of considering a fractional integro-differential operator in a feedback loop, though without using the term "fractional", was made by Bode (1940), and next in a more comprehensive way in Bode (1945). A key problem in the design of a feedback amplifier was to come up with a feedback loop so that the performance of the closed loop was invariant to changes in the amplifier gain. Bode presented an elegant solution to this robust design problem, which he called the ideal cutoff characteristic, nowadays known as ideal loop transfer function, whose Nyquist plot is a straight line through the origin giving a phase margin invariant to gain changes. Clearly, this ideal system is a fractional integrator with transfer function $G(s)=\left(\omega_{c g} / s\right)^{\alpha}$, known as Bode's ideal transfer function, where $\omega_{c g}$ is the gain crossover frequency and the constant phase margin is $\varphi_{m}=\pi-\alpha \pi / 2$. This frequency characteristic is very interesting in terms of robustness of the system to parameters changes or uncertainties, and several design methods make use of it. In fact, the fractional integrator can be used as an alternative reference system for control, considering its 
own properties (Vinagre, Monje, Calderón, Chen, \& Feliu, 2004).

This first step toward the application of fractional calculus in control led to the adaptation of the FC concepts to frequency-based methods. The frequency response and the transient response of the non-integer integral (in fact Bode's ideal transfer function) and its application to control systems were introduced by Manabe (1961), and more recently in Barbosa, Tenreiro, and Ferreira (2003).

Going a step further in automatic control, Oustaloup (1991) studied the fractional order algorithms for the control of dynamic systems and demonstrated the superior performance of the CRONE (Commande Robuste d'Ordre Non Entier) method over the PID controller. There are three generations of CRONE controllers, and Oustaloup, Levron, Nanot, and Mathieu (2000) concentrate on the third generation. Podlubny (1999b) proposed a generalization of the $P I D$ controller, namely the $P I^{\lambda} D^{\mu}$ controller, involving an integrator of order $\lambda$ and a differentiator of order $\mu$. He also demonstrated the better response of this type of controller, in comparison with the classical PID controller, when used for the control of fractional order systems. A frequency domain approach by using fractional order PID controllers was also studied in Vinagre, Podlubny, Dorčák, and Feliu (2000).

Further research activities run in order to define new effective tuning techniques for non-integer order controllers by an extension of the classical control theory. To this respect, in Caponetto, Fortuna, and Porto $(2002,2004)$ the extension of derivation and integration orders from integer to non-integer numbers provides a more flexible tuning strategy and therefore an easier achieving of control requirements with respect to classical controllers. In Leu, Tsay, and Hwang (2002) an optimal fractional order PID controller based on specified gain and phase margins with a minimum integral squared error (ISE) criterion is designed. Other works (Vinagre, 2001; Vinagre, Monje, \& Calderón, 2002) take advantage of the fractional orders introduced in the control action in order to design a more effective controller to be used in real-life models (see also Chen, 2006). The tuning of integer PID controllers is addressed in Barbosa, Tenreiro, and Ferreira $(2003,2004 a, 2004 b)$ by minimizing a penalty function that reflects how far the behavior of the PID is from that of a desired fractional transfer function, and in Chen, Moore, Vinagre, and Podlubny (2004) and Chen, Vinagre, and Podlubny (2004) with a somewhat similar strategy. Another approach is the use of a new control strategy to control first-order systems with long time delay (Chen, Vinagre, \& Monje, 2003; Monje, Calderón, \& Vinagre, 2002). A robustness constraint is considered in this last work, forcing the phase of the open-loop system to be flat at the gain crossover frequency.

Fractional calculus also extends to other kinds of control strategies different from PID ones. In what concerns $\mathrm{H}_{2}$ and $H_{\infty}$ controllers, for instance, Malti, Aoun, Cois, Oustaloup, and Levron (2003) discuss the reckoning of the
$\mathrm{H}_{2}$ norm of a fractional SISO system (without applying the result to the development of controllers), and Petráš and Hypiusova (2002) suggest the tuning of $H_{\infty}$ controllers for fractional SISO systems by numerical minimization.

Applications of fractional calculus in control are numerous. In Yago Sánchez (1999) the control of viscoelastic damped structures is aimed. Control applications to a flexible transmission (Oustaloup, Mathieu, \& Lanusse, 1995; Valério, 2001), an active suspension (Lanusse, Poinot, Cois, Oustaloup, \& Trigeassou, 2003), a buck converter (Calderón, 2003; Calderón, Vinagre, \& Feliu, 2003) and a hydraulic actuator (Pommier, Musset, Lanusse, \& Oustaloup, 2003) are found in the literature. The fractional control of rigid robots is the objective in Fonseca and Tenreiro (2003), Tenreiro and Azenha (1998), and the fractional control of a thermal system is the objective in Sabatier and Oustaloup (2003), Petráš and Vinagre (2002), Petráš, Vinagre, Dorčák, and Feliu (2002), Vinagre, Petráš, Merchán, and Dorčák (2001). Besides, other applications such as the robust control of main irrigation canals (Feliu, Rivas, \& Sánchez, 2007) and robustness analysis of a winding system (Laroche \& Knittel, 2005) can be found.

Regarding the implementation of fractional order controllers, a very good review is given in Valério (2005) referring to continuous and discrete approximations of fractional order systems. Other related references are Chen and Moore (2002), Monje (2006), Oustaloup et al. (2000), Podlubny, Petráš, Vinagre, O'Leary, and Dorčák (2002), Vinagre, Podlubny, Dorčák et al. (2000), Chen, Moore et al. (2004), and Chen, Vinagre et al. (2004).

To sum all this up, it is clear that FOC and its applications are becoming an important issue. Of course, there are other published texts related to fractional calculus. The main reason why they are not cited here is that their subjects are not relevant for the purpose of this work.

\subsection{Implementation of fractional order controllers}

Before introducing the essentials of the design method for the fractional order $P I^{\lambda} D^{\mu}$ controller, some initial considerations on its implementation have to be taken into account.

The generalized transfer function of this controller is given by

$C(s)=k_{p}+\frac{k_{i}}{s^{\lambda}}+k_{d} s^{\mu}$.

Next statements are important to be considered. First of all, properly implemented, a fractional integrator of order $k+\alpha, k \in N, 0<\alpha<1$, is, for steady-state error cancellation, as efficient as an integer order integrator of order $k+1$ (see Axtell \& Bise, 1990). However, though the final value theorem states that the fractional system exhibits null steady-state error if $\alpha>0$, the fact of being $\alpha<1$ makes the output converge to its final value more slowly than in the 
case of an integer controller. Furthermore, the fractional effect has to be band-limited when it is implemented. Therefore, the fractional integrator must be implemented as $1 / s^{\alpha}=(1 / s) s^{1-\alpha}$, ensuring this way the effect of an integer integrator $1 / s$ at very low frequency.

Similarly to the fractional integrator, the fractional differentiator, $s^{\mu}$, has also to be band-limited when implemented, ensuring this way a finite control effort and noise rejection at high frequencies.

On the other hand, when fractional order controllers have to be implemented or simulations have to be performed, fractional transfer functions are usually replaced by integer transfer functions with a behavior close enough to the one desired, but much easier to handle. There are many different ways of finding such approximations but unfortunately it is not possible to say that one of them is the best, because even though some of them are better than others in regard to certain characteristics, the relative merits of each approximation depend on the differentiation order, on whether one is more interested in an accurate frequency behavior or in accurate time responses, on how large admissible transfer functions may be, and other factors like these. A good review of these approximations can be found in Valério (2005), Vinagre, Podlubny, Hernández, and Feliu (2000).

In this work two different ways to approximate fractional order operators to an integer transfer function have been used: the Oustaloup continuous approximation (Oustaloup et al., 2000; Oustaloup, 1995) and a frequency identification method performed by the Matlab function invfreqs (MathWorks, 2000b). With both methods a rational transfer function is obtained whose frequency response fits the frequency response of the original irrational transfer function. These two methods are chosen due to their accuracy in the frequency range of interest, and any other of the techniques in Valério (2005), Vinagre, Podlubny, Hernández et al. (2000) could also be suitable for that purpose.

Once a continuous approximation of the fractional order operator is obtained, and for the sake of implementation, the Tustin method with prewarping (Levine, 1996) has been applied in this work for the discretization of the resulting approximation.

\section{A tuning method for fractional order $P I^{\lambda} D^{\mu}$ controllers}

\subsection{Design specifications and tuning problem}

As commented in the introduction, the objective of this paper is to design a fractional order controller so that the system fulfills different specifications regarding robustness to plant uncertainties, load disturbances and high frequency noise. For that reason, specifications related to phase margins, sensitivity functions and robustness constraints are going to be considered in this design method, due to their important features regarding performance, stability and robustness. Of course, other kinds of specifications can be met, depending on the particular requirements of the system. Therefore, the design problem is formulated as follows:

- Phase margin $\left(\varphi_{m}\right)$ and gain crossover frequency $\left(\omega_{c g}\right)$ specifications: Gain and phase margins have always served as important measures of robustness. It is known that the phase margin is related to the damping of the system and therefore can also serve as a performance measure (see Franklin, Powell, \& Naeini, 1986). The equations that define the phase margin and the gain crossover frequency are

$$
\begin{aligned}
& \left|C\left(\mathrm{j} \omega_{c g}\right) G\left(\mathrm{j} \omega_{c g}\right)\right|_{\mathrm{dB}}=0 \mathrm{~dB}, \\
& \arg \left(C\left(\mathrm{j} \omega_{c g}\right) G\left(\mathrm{j} \omega_{c g}\right)\right)=-\pi+\varphi_{m} .
\end{aligned}
$$

- Robustness to variations in the gain of the plant: The next constraint can be considered in this case (see Chen \& Moore, 2005):

$$
\left(\frac{\mathrm{d}(\arg (F(s)))}{\mathrm{d} \omega}\right)_{\omega=\omega_{c g}}=0 .
$$

This condition forces the phase of the open-loop system $F(s)=C(s) G(s)$ to be flat at $\omega_{c g}$ and hence to be almost constant within an interval around $\omega_{c g}$. It means that the system is more robust to gain changes and the overshoot of the response is almost constant within a gain range (iso-damping property of the time response). It must be remarked that the interval of gains for which the system is robust is not fixed with this condition. That is, the user cannot force the system to be robust for a particular gain range. This range depends on the frequency range around $\omega_{c g}$ for which the phase of the open-loop system keeps flat. This frequency range will be longer or shorter, depending on the resulting controller and the plant.

- High frequency noise rejection: A constraint on the complementary sensitivity function $\mathrm{T}$ can be established:

$\left|T(\mathrm{j} \omega)=\frac{C(\mathrm{j} \omega) G(\mathrm{j} \omega)}{1+C(\mathrm{j} \omega) G(\mathrm{j} \omega)}\right|_{\mathrm{dB}} \leqslant A \mathrm{~dB}$,

$\forall \omega \geqslant \omega_{t} \mathrm{rad} / \mathrm{s} \Rightarrow\left|T\left(\mathrm{j} \omega_{t}\right)\right|_{\mathrm{dB}}=A \mathrm{~dB}$

whit $A$ the desired noise attenuation for frequencies $\omega \geqslant \omega_{t} \mathrm{rad} / \mathrm{s}$.

- To ensure a good output disturbance rejection: A constraint on the sensitivity function $S$ can be defined:

$\left|S(\mathrm{j} \omega)=\frac{1}{1+C(\mathrm{j} \omega) G(\mathrm{j} \omega)}\right|_{\mathrm{dB}} \leqslant B \mathrm{~dB}$,

$\forall \omega \leqslant \omega_{s} \mathrm{rad} / \mathrm{s} \Rightarrow\left|S\left(\mathrm{j} \omega_{s}\right)\right|_{\mathrm{dB}}=B \mathrm{~dB}$

with $B$ the desired value of the sensitivity function for frequencies $\omega \leqslant \omega_{s} \mathrm{rad} / \mathrm{s}$ (desired frequency range).

- Steady-state error cancellation: As stated before, the fractional integrator $s^{-\lambda}$ is, for steady-state error cancellation, as efficient as an integer order integrator. So, the specification of null steady state-error is fulfilled 
with the introduction of the fractional integrator, properly implemented.

Using the fractional order $P I^{\lambda} D^{\mu}$ controller of Eq. (5), up to five of these design specifications can be fulfilled, since it has five parameters to tune. For fractional order controllers such as a $P I^{\lambda}$ or a $P D^{\mu}$, three design specifications could be met (one for each parameter). Therefore, for the general case of a $P I^{\lambda} D^{\mu}$ controller the design problem is based on solving the system of five nonlinear equations (given by the corresponding design specifications) and five unknown parameters $k_{p}, k_{d}, k_{i}, \lambda, \mu$.

However, the complexity of this set of nonlinear equations is very significant, specially when fractional orders of the Laplace variable $s$ are introduced, and finding out the solution is not trivial. In fact, a nonlinear optimization problem must be solved, in which the best solution of a constrained nonlinear equation has to be found.

Global optimization is the task of finding the absolutely best set of admissible conditions to achieve an objective under given constraints, assuming that both are formulated in mathematical terms. Some large-scale global optimization problems have been solved by current methods, and a number of software packages are available that reliably solve most global optimization problems in small (and sometimes larger) dimensions. However, finding the global minimum, if one exists, can be a difficult problem (very dependant on the initial conditions). Superficially, global optimization is a stronger version of local optimization, whose great usefulness in practice is undisputed. Instead of searching for a locally feasible point one wants the globally best point in the feasible region. However, in many practical applications finding the globally best point, though desirable, is not essential, since any sufficiently good feasible point is useful and usually an improvement over what is available without optimization (this particular case). Besides, sometimes, depending on the optimization problem, there is no guarantee that the optimization functions will return a global minimum, unless the global minimum is the only minimum and the function to minimize is continuous (Pintér, 1996). Taking all these into account, and considering that the set of functions to minimize in this case is continuous and can only present one minimum in the feasible region, any of the optimization methods available could be effective, a priori. For this reason, and taking into account that Matlab is a very appropriate tool for the analysis and design of control systems, the optimization toolbox of Matlab has been used to reach out the best solution with the minimum error. The function used for this purpose is called FMINCON (MathWorks, 2000a), which finds the constrained minimum of a function of several variables. It solves problems of the form $\mathrm{MIN}_{X} \mathrm{~F}(X) \quad$ subject $\quad$ to: $\quad C(X) \Leftarrow 0, \quad C_{e q}(X)=0$, $L B \Leftarrow X \Leftarrow U B$, where $F$ is the function to minimize; $C$ and $C_{e q}$ represent the nonlinear inequalities and equalities, respectively (nonlinear constraints); $X$ is the minimum looked for; $L B$ and $U B$ define a set of lower and upper bounds on the design variables, $X$.

In this particular case, the specification in Eq. (6) is taken as the main function to minimize, and the rest of specifications ((7)-(10)) are taken as constrains for the minimization, all of them subjected to the optimization parameters defined within the function FMINCON. The success of this design method depends mainly on the initial conditions considered for the parameters of the controller. In Section 4 a different tuning method for this kind of controller is proposed in which only the frequency characteristics of the plant at some frequencies of interest is enough for the tuning purpose, without considering initial conditions for the parameters and avoiding the nonlinear minimization problem.

The tuning method proposed here is illustrated next with the results obtained from an experimental platform consisting on a liquid level system.

\subsection{Experimental results by using the tuning method}

The experimental platform Basic Process Rig 38-100 Feedback Unit has been used to test the fractional order controllers designed by the optimization tuning method proposed previously. The platform consists on a low pressure flowing water circuit which is bench mounted and completely self contained. The water circuit is arranged in front of a vertical panel, as can be seen in Fig. 1.

For the characterization of the plant and implementation of the controller a data acquisition board PCL-818H, by PC-LabCard, has been used, running on Matlab 5.3 and using its real time toolbox "Real-Time Windows Target". A computer Pentium II, $350 \mathrm{MHz}, 64 \mathrm{M}$ RAM, supports the data acquisition board and the program in $C$ programming language (from Matlab) corresponding to the controller.

After the characterization of the system the resulting transfer function is

$G(s)=\frac{k}{\tau s+1} \mathrm{e}^{-L s}=\frac{3.13}{433.33 s+1} \mathrm{e}^{-50 s}$,

that is, the liquid level system is modeled by a first-order transfer function with time delay $L=50 \mathrm{~s}$, gain $k=3.13$ and time constant $\tau=433.33 \mathrm{~s}$. The design specifications required for the system are:

- gain crossover frequency, $\omega_{c g}=0.008 \mathrm{rad} / \mathrm{s}$;

- phase margin, $\varphi_{m}=60^{\circ}$;

- robustness to variations in the gain of the plant must be fulfilled;

- sensitivity function: $|S(\mathrm{j} \omega)|_{\mathrm{dB}} \leqslant-20 \mathrm{~dB}, \quad \forall \omega \leqslant \omega_{s}=$ $0.001 \mathrm{rad} / \mathrm{s}$;

- noise rejection: $|T(\mathrm{j} \omega)|_{\mathrm{dB}} \leqslant-20 \mathrm{~dB}, \forall \omega \geqslant \omega_{t}=10 \mathrm{rad} / \mathrm{s}$.

Applying the optimization method described previously, the fractional $P I^{\lambda} D^{\mu}$ controller obtained to control the 


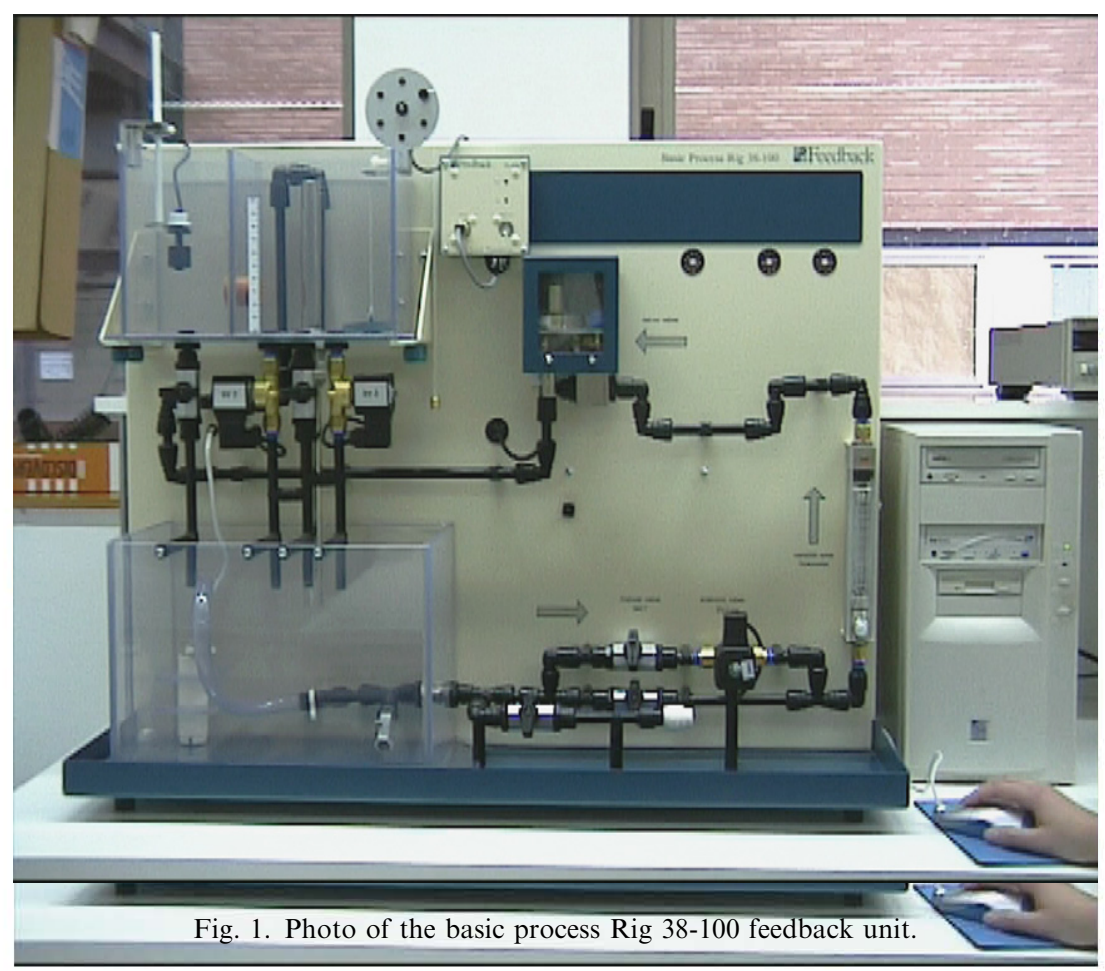

system is

$C(s)=0.6152+\frac{0.0100}{s^{0.8968}}+4.3867 s^{0.4773}$.

In this particular case the fractional integral and derivative parts have been implemented by the Oustaloup continuous approximation of the fractional integrator (Oustaloup, 1995; Oustaloup et al., 2000), choosing a frequency band from 0.001 to $100 \mathrm{rad} / \mathrm{s}$ and an order of the approximation equal to 5 (number of poles and zeros). Once the continuous fractional controller is obtained, it is discretized by using the Tustin rule with a sampling time $T_{s}=1 \mathrm{~s}$ and a prewarp frequency $\omega_{c g}$ (Levine, 1996).

The Bode plots of the open-loop system $F(s)=C(s) G(s)$ are shown in Fig. 2. As can be observed, specifications of gain crossover frequency and phase margin are met. Besides, the phase of the system is forced to be flat at $\omega_{c g}$ and hence to be almost constant within an interval around $\omega_{c g}$. It means that the system is more robust to gain changes and the overshoot of the response is almost constant within this interval, as can be seen in Fig. 3, where a step input of 0.47 has been applied to the closed-loop system. Variations in the gain of the plant have been considered from 2.75 to 3.75 . The magnitudes of the functions $S(s)$ and $T(s)$ for the nominal plant are shown in Figs. 4 and 5, respectively, fulfilling the specifications.

The experimental results obtained when controlling the liquid level plant in real time are shown next. Fig. 6 shows the comparison between simulated and experimental levels for the nominal gain $k=3.13$. In Fig. 7 the experimental responses for different gains (set by software) are scoped, fulfilling the robustness constraint to gain changes (within the variation range selected). Fig. 8 shows the experimental
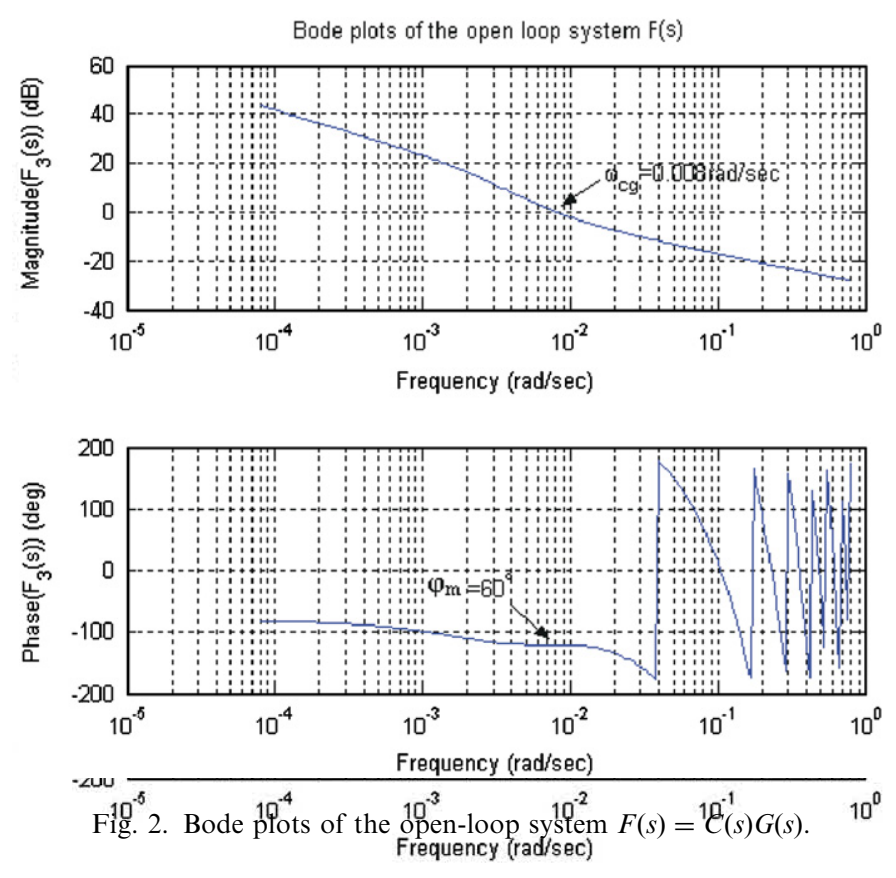

control laws obtained for each value of gain. As far as the control laws are concerned, only a slight variation in the peak value of the signal is produced when the gain changes, which is an important feature as far as the saturation of the actuator is concerned. In this case, the peak value is very far from the saturation value of $10 \mathrm{~V}$ for the servo valve.

From these results, the potential of the fractional order controllers in practical industrial settings, regarding performance and robustness aspects, is clear. However, the design method proposed here involves complex equations relating 


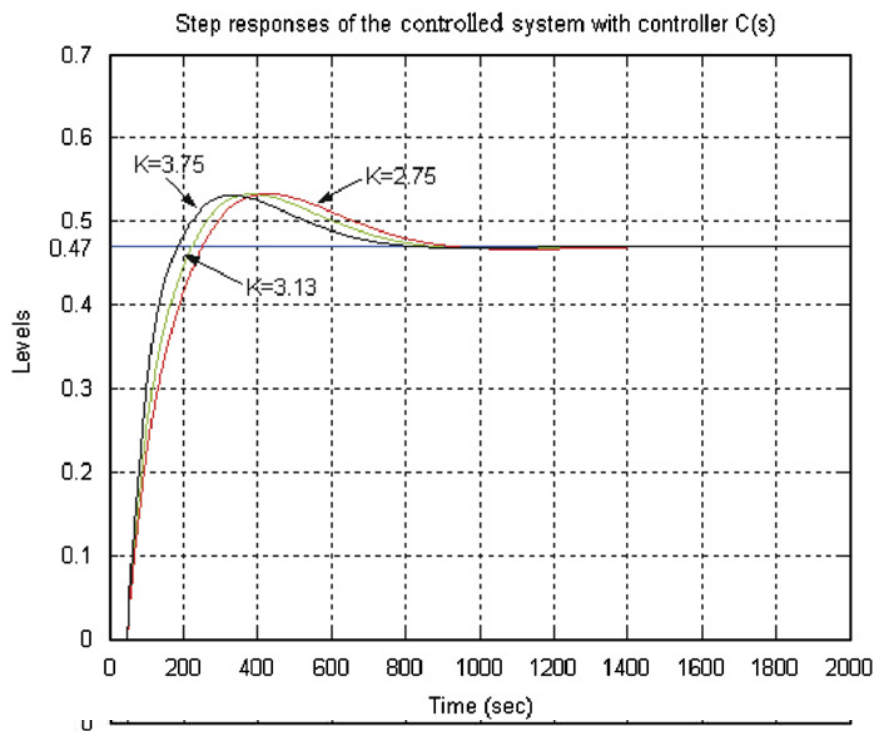

Fig. 3. Simulation step responses of the controfled system with controller $C(s)$.
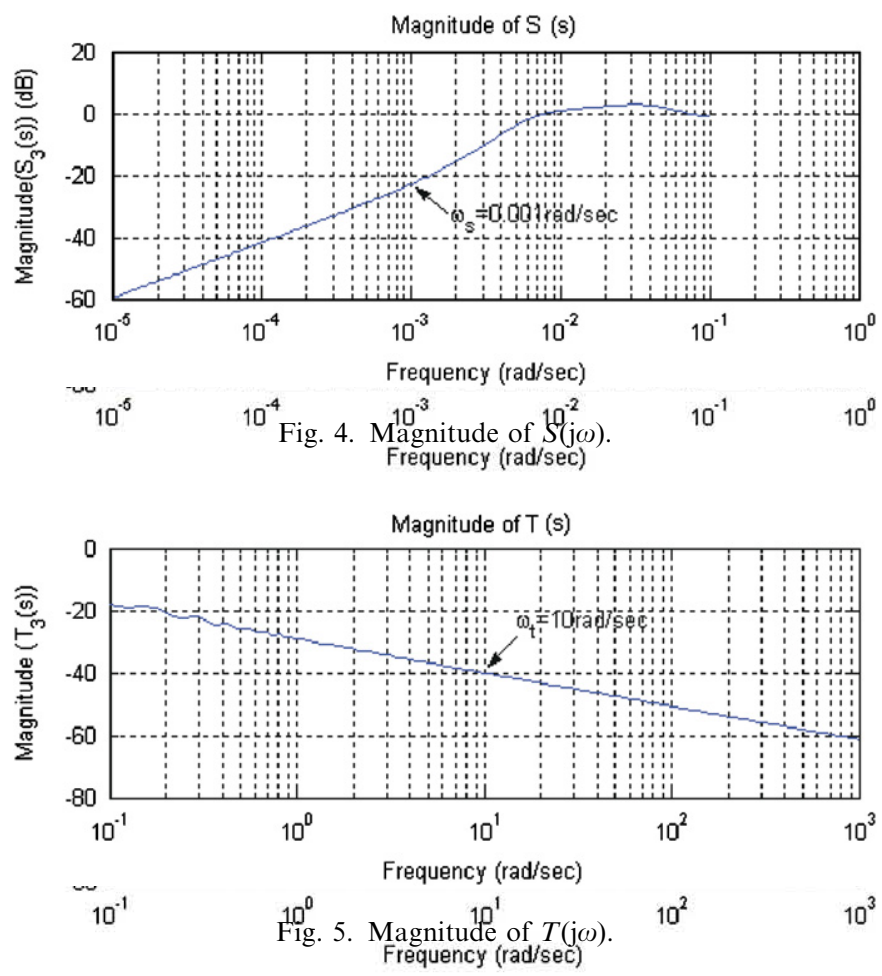

the specifications of design and, sometimes, it may be difficult to find a solution to the problem. For this reason, the purpose now is to simplify the design method so that the controller can be tuned very easily, with very simple relations among its parameters, and preserving the robustness characteristics regarding performance, gain variations and noise. Besides, this new method will allow the automatic tuning (auto-tuning) of the fractional order controller without the need of knowing the plant model (its transfer function). The relay test will be used for that purpose, as will be described next.

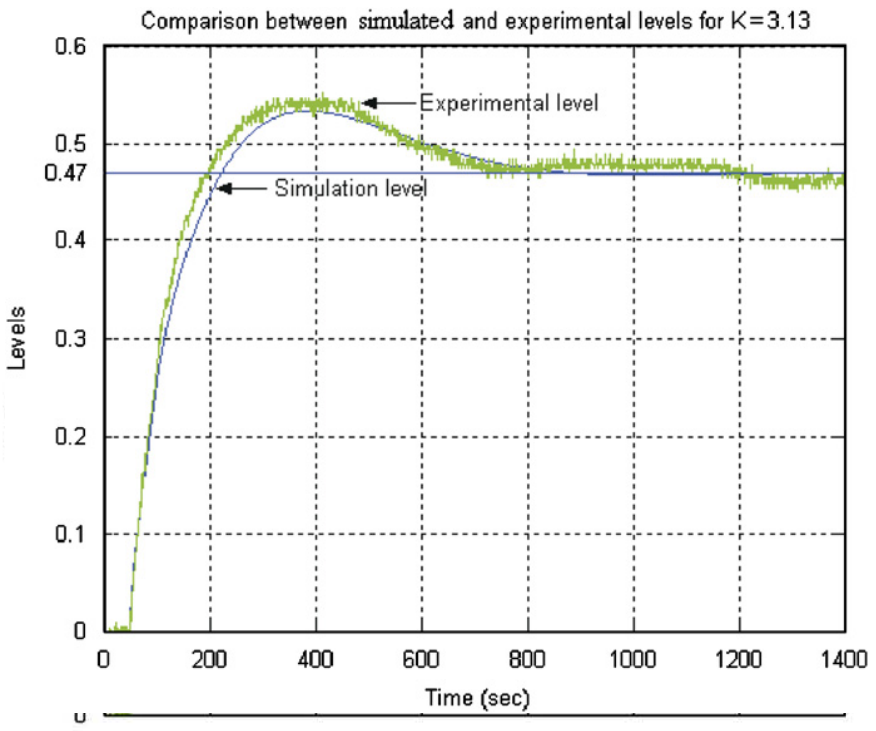

Fig. 6. Comparison between simulated and experimental levels for ${ }^{14000}=$ 3.13 and controller $C(s)$.

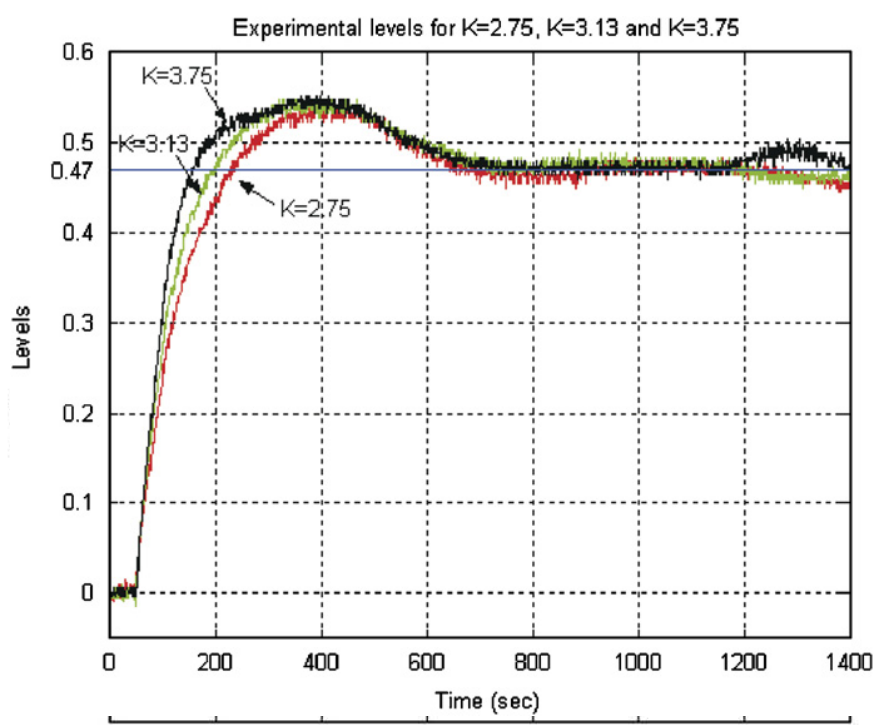

Fig. 7. Experimental step responses of ${ }^{2000}$ the controlled 1200 system 1400 controller $C(s)$.

\section{Auto-tuning of fractional order controllers}

Many process control problems can be adequately and routinely solved by conventional PID-control strategies. The reason why the PID controller is so widely accepted is its simple structure, which has proven to be appropriate for many commonly met control problems such as setpoint regulation/tracking, disturbance attenuation, and the like. However, although tuning guidelines are available, the tuning process can still be time consuming with the result that many control loops are often poorly tuned and full potential of the control system is not achieved. These methods require a fair amount of a priory knowledge as, for instance, sampling time, dead time, model order, and desired time response. This knowledge may either be given 


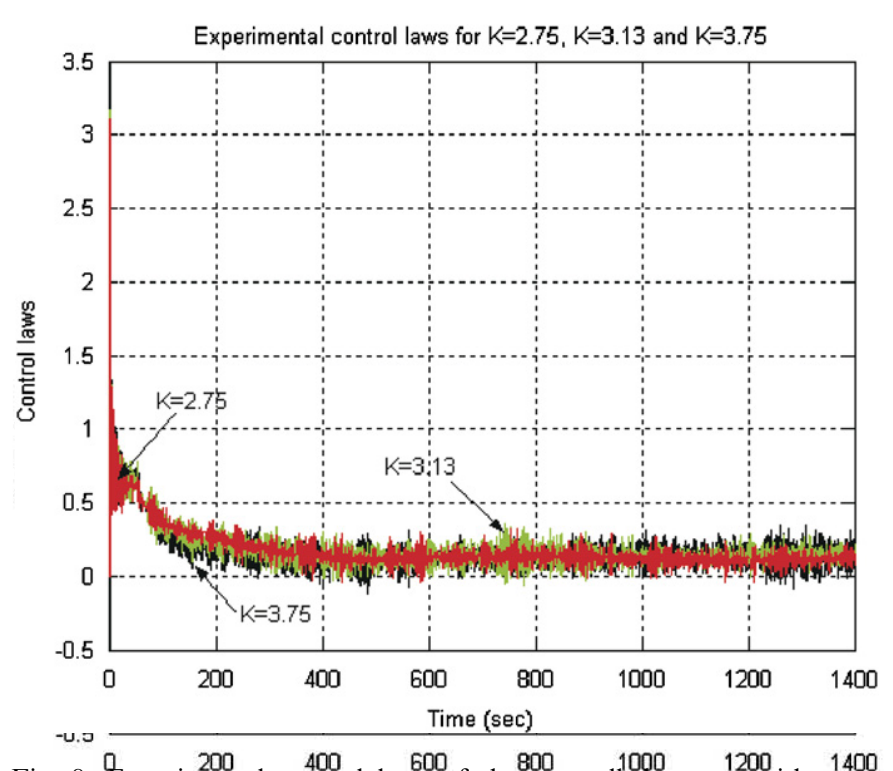

Fig. 8. Experimental control laws of the controlled system with controller $C(s)$

by a skilled engineer or may be acquired automatically by some kind of experimentation. The second alternative, commonly known as auto-tuning, is preferable not only to simplify the task of the operator but also for the sake of robustness.

There are a wide variety of auto-tuning methods for integer controllers. Some of them aim in someway the robustness of the controlled system (see Tan, Huang, \& Ferdous, 2002), for example, forcing the phase of the openloop system to be flat around the crossover frequency so that the system is robust to gain variations (see Chen \& Moore, 2005; Chen, Moore et al., 2004; Chen, Vinagre et al., 2004). However, the complexity of the equations relating the parameters of the controller increases when some kinds of robustness constraints are required for the controlled system. The implementation of these types of auto-tuning methods for industrial purposes will be really complicate since, in general, industrial devices such as a PLC cannot solve sets of complex nonlinear equations.

For that reason, an auto-tuning method for fractional order $P I^{\lambda} D^{\mu}$ controllers based on the relay test is proposed, that allows the fulfilment of robustness constrains for the controlled system by simple relations among the parameters of the controller, simplifying the later implementation process.

The final aim is to find out a method to auto-tune a fractional order $P I^{\lambda} D^{\mu}$ controller formulated as

$C(s)=k_{c} x^{\mu}\left(\frac{\lambda_{1} s+1}{s}\right)^{\lambda}\left(\frac{\lambda_{2} s+1}{x \lambda_{2} s+1}\right)^{\mu}$.

As can be observed, this controller has two different parts given by the following equations:

$P I^{\lambda}(s)=\left(\frac{\lambda_{1} s+1}{s}\right)^{\lambda}$,
$P D^{\mu}(s)=k_{c} x^{\mu}\left(\frac{\lambda_{2} s+1}{x \lambda_{2} s+1}\right)^{\mu}$.

Eq. (14) corresponds to a fractional order $P I^{\lambda}$ controller and Eq. (15) to a fractional order lead compensator that can be identified as a $P D^{\mu}$ controller plus a noise filter. In this method, the fractional order $P I^{\lambda}$ controller will be used to cancel the slope of the phase of the plant at the gain crossover frequency $\omega_{c g}$. This way, a flat phase around the frequency of interest is ensured. Once the slope is cancelled, the $P D^{\mu}$ controller will be designed to fulfill the design specifications of gain crossover frequency, $\omega_{c g}$, and phase margin, $\varphi_{m}$, following a robustness criterion based on the flatness of the phase curve of this compensator, as will be explained later. This way, the resulting phase of the openloop system will be the flattest possible, ensuring the maximum robustness to plant gain variations.

Let us firstly give some remarks about the relay test used for the auto-tuning problem.

\subsection{Relay test for auto-tuning}

The relay auto-tuning process has been widely used in industrial applications (see Hang, Åström, \& Wang, 2002). The choice of relay feedback to solve the design problem is justified by the possible integration of system identification and control into the same design strategy, giving birth to relay auto-tuning. In this work a variation of the standard relay test is used, shown in Fig. 9, where a delay $\theta_{a}$ is introduced after the relay function. With this scheme, as explained in Chen and Moore (2005), the next relations are given:

$\arg \left(G\left(\mathrm{j} \omega_{c}\right)\right)=-\pi+\omega_{c} \theta_{a}$,

$\left|G\left(\mathrm{j} \omega_{c}\right)\right|=\frac{\pi a}{4 d}=\frac{1}{N(a)}$,

where $G\left(\mathrm{j} \omega_{c}\right)$ is the transfer function of the plant at the frequency $\omega_{c}$, which is the frequency of the output signal $y$ corresponding to the delay $\theta_{a}, d$ is the relay output, $a$ is the amplitude of the output signal (signal " $y$ " in Fig. 9), and $N(a)$ is the equivalent relay gain. This way, for each value of $\theta_{a}$ a different point on the Nyquist curve of the plant is obtained. Therefore, a point on the Nyquist curve of the plant at a particular desired frequency $\omega_{c}$ can be identified,

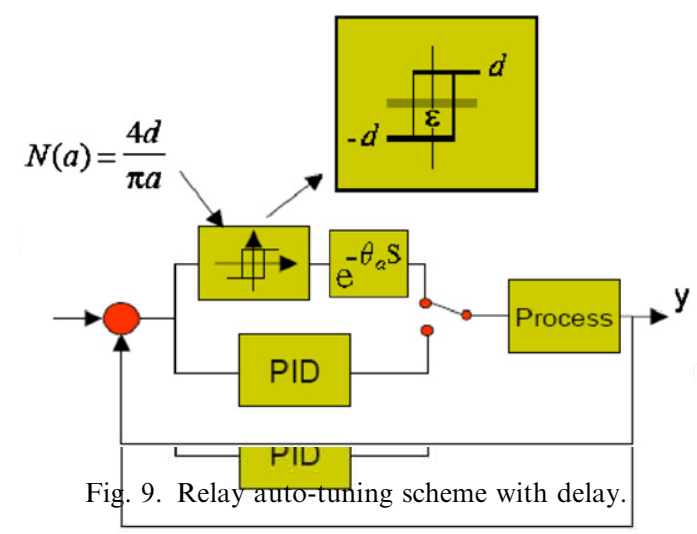


for example, at the gain crossover frequency required for the controlled system $\left(\omega_{c}=\omega_{c g}\right)$. The problem would be how to select the right value of $\theta_{a}$ which corresponds to a specific frequency $\omega_{c}$. An iterative method can be used to solve this problem, as presented in Chen and Moore (2005). The artificial time delay parameter can be updated using the simple interpolation/extrapolation scheme $\theta_{n}=$ $\left(\omega_{c}-\omega_{n-1}\right) /\left(\omega_{n-1}-\omega_{n-2}\right)\left(\theta_{n-1}-\theta_{n-2}\right)+\theta_{n-1}$, where $n$ represents the current iteration number. With the new $\theta_{n}$, after the relay test, the corresponding frequency $\omega_{n}$ can be recorded and compared with the frequency $\omega_{c}$ so that the iteration can continue or stop. Two initial values of the delay $\left(\theta_{-1}\right.$ and $\left.\theta_{0}\right)$ and their corresponding frequencies $\left(\omega_{-1}\right.$ and $\left.\omega_{0}\right)$ are needed to start the iteration. Therefore, first of all, a value for $\theta_{-1}$ is selected and the relay test is carried out, obtaining an output signal with frequency $\omega_{-1}$. Then, in a second iteration, another value is given for $\theta_{0}$, obtaining an output signal with frequency $\omega_{0}$. With these two pairs $\left(\theta_{-1}, \omega_{-1}\right)$ and $\left(\theta_{0}, \omega_{0}\right)$ the next value of $\theta_{n}$ is automatically obtained by using the interpolation/extrapolation scheme above.

Let us now concentrate on the design of the fractional order $P I^{\lambda}$ controller.

\subsection{Design of the fractional order $P I^{\lambda}$ controller}

The fractional order $P I^{\lambda}$ controller of Eq. (14) will be used to cancel the slope of the phase of the plant in order to obtain a flat phase around the frequency point $\omega_{c g}$. The value of this slope is given by expression

$v=\frac{\phi_{u}-\phi_{n-1}}{\omega_{u}-\omega_{n-1}} \frac{\mathrm{rad}}{\mathrm{rad} / \mathrm{s}}$

where $\omega_{n-1}$ is the frequency $n-1$ experimented with the relay test and $\phi_{n-1}$ its corresponding plant phase, and $\phi_{u}$ the plant phase corresponding to the frequency of interest $\omega_{u}=\omega_{c g}$.

The phase of the fractional order $P I^{\lambda}$ controller is given by

$\psi=\arg \left(P I^{\lambda}(s)\right)=\lambda\left(\arctan \left(\lambda_{1} \omega\right)-\pi / 2\right)$.

In order to cancel the slope of the phase curve of the plant, $v$, the derivative of the phase of $P I^{\lambda}(s)$ at the frequency point $\omega_{c g}$ must be equal to $-v$, resulting the equation:

$\psi^{\prime}=\left[\frac{\mathrm{d} \psi}{\mathrm{d} \omega}\right]_{\omega=\omega_{c g}}=\lambda \frac{\lambda_{1}}{1+\left(\lambda_{1} \omega_{c g}\right)^{2}}=-v$.

The parameters $\lambda$ and $\lambda_{1}$ must be selected so that this expression is fulfilled. Studying the function (20) and differentiating with respect to parameter $\lambda_{1}$ (see Eq. (21)), it is obtained that it has a maximum at $\lambda_{1}=1 / \omega_{c g}$ (see Eq. (22)), as can be observed in Fig. 10.

$\frac{\mathrm{d} \psi^{\prime}}{\mathrm{d} \lambda_{1}}=\lambda\left(\frac{\left(\lambda_{1} \omega_{c g}\right)^{2}-1}{\left(1+\left(\lambda_{1} \omega_{c g}\right)^{2}\right)^{2}}\right)$,

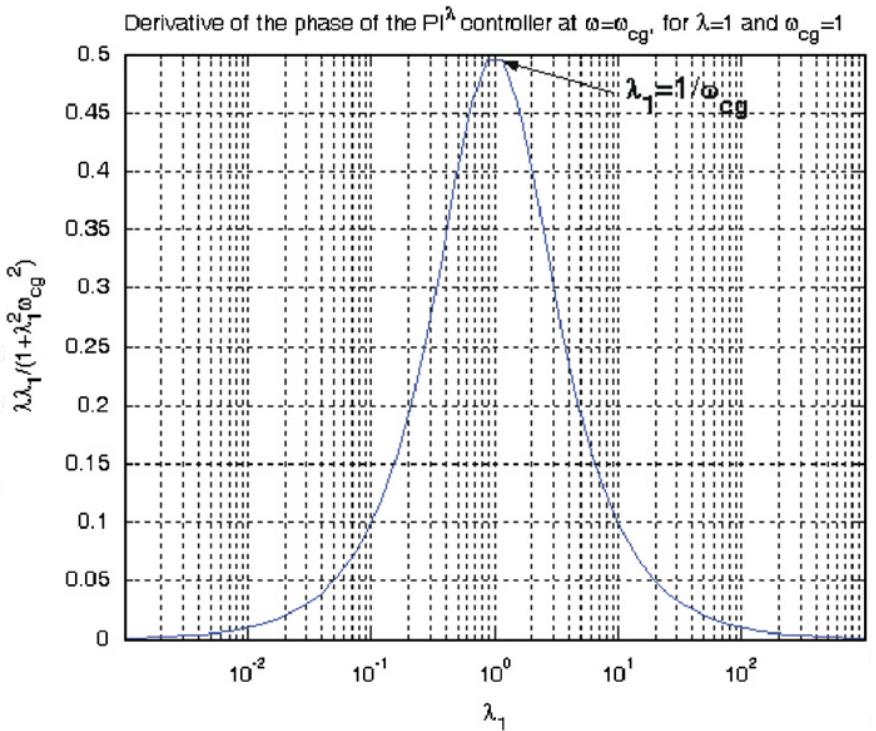
Fig. 10. Derivative of the phase of the $P I^{10^{-2}} \lambda_{1}^{10}$ controller at $\omega=\omega_{c g}^{10^{2}}$, for $\lambda=1$
and $\omega_{c g}=1$.

$\frac{\mathrm{d} \psi^{\prime}}{\mathrm{d} \lambda_{1}}=0 \Rightarrow\left(\lambda_{1} \omega_{c g}\right)^{2}-1=0 \Rightarrow \lambda_{1}=\frac{1}{\omega_{c g}}$

That is, choosing $\omega_{\text {cero }}=1 / \lambda_{1}=\omega_{c g}$ the slope of the plant at the frequency $\omega_{c g}$ will be cancelled with the maximum slope of the fractional order controller. Once the value of $\lambda_{1}$ is fixed, the value of $\lambda$ is easily determined by $\lambda=\left(-v\left(1+\left(\lambda_{1} \omega_{c g}\right)^{2}\right)\right) / \lambda_{1}$. It is observed that the value of $\lambda$ obtained will be minimum when $\lambda_{1}=1 / \omega_{c g}$. Variations of the frequency $\omega_{\text {cero }}$ up or down the frequency $\omega_{c g}$ will produce higher values of the parameter $\lambda$. Therefore, selecting $\omega_{\text {cero }}=\omega_{c g}$ the phase lag of the resulting $P I^{\lambda}(s)$ controller will be the minimum one (minimum $\lambda$ ). This fact is very interesting from the robustness point of view. The less the phase lag of the controller $P I^{\lambda}(s)$, the less the phase lead of the controller $P D^{\mu}(s)$ at the frequency $\omega_{c g}$, favoring the flatness of its phase curve. Then, considering this robustness criterion, the value of $\lambda_{1}$ will be fixed to $1 / \omega_{c g}$. Remember that the real value of $\omega_{c g}$ to be used in the design is $\omega_{u}$, which is the one obtained with the relay test and very close to $\omega_{c g}$.

\subsection{Design of the fractional order $P D^{\mu}$ controller}

Defining the system $G_{\text {flat }}(s)=G(s) P I^{\lambda}(s)$, now the controller $P D^{\mu}(s)$ will be designed so that the open-loop system $F(s)=G_{\text {flat }}(s) P D^{\mu}(s)$ fulfills the specifications of gain crossover frequency, $\omega_{c g}$, and phase margin, $\varphi_{m}$, following a robustness criterion based on the flatness of the phase curve of this compensator, as will be explained next (Monje, Calderón, Vinagre, Chen, \& Feliu, 2004; Monje, Vinagre, Calderón, Feliu, \& Chen, 2005).

For a specified phase margin, $\varphi_{m}$, and gain crossover frequency, $\omega_{c g}$, the following relationships for the open-loop 
system can be given in the complex plane:

$$
\begin{aligned}
& G_{f l a t}\left(\mathrm{j} \omega_{c g}\right) \cdot k^{\prime}\left(\frac{\mathrm{j} \lambda_{2} \omega_{c g}+1}{\mathrm{j} x \lambda_{2} \omega_{c g}+1}\right)^{\mu}=\mathrm{e}^{\mathrm{j}\left(-\pi+\varphi_{m}\right)} \\
& \quad \Rightarrow C^{\prime}\left(\mathrm{j} \omega_{c g}\right)=\left(\frac{\mathrm{j} \lambda_{2} \omega_{c g}+1}{\mathrm{j} x \lambda_{2} \omega_{c g}+1}\right)^{\mu}=\frac{\mathrm{e}^{\mathrm{j}\left(-\pi+\varphi_{m}\right)}}{G_{f l a t}\left(\mathrm{j} \omega_{c g}\right) \cdot k^{\prime}}=a_{1}+\mathrm{j} b_{1} \\
& \quad \Rightarrow\left(\frac{\mathrm{j} \lambda_{2} \omega_{c g}+1}{\mathrm{j} x \lambda_{2} \omega_{c g}+1}\right)=\left(a_{1}+\mathrm{j} b_{1}\right)^{1 / \mu}=a+\mathrm{j} b
\end{aligned}
$$

where $k^{\prime}=k_{c} x^{\mu}=1$ in this case, $G_{\text {flat }}(s)$ is the plant to be controlled, and $\left(a_{1}, b_{1}\right)$ is called the "design point". Parameter $x$ sets the distance between the zero $\left(1 / \lambda_{2}\right)$ and pole $\left(1 / x \lambda_{2}\right)$ of the $P D^{\mu}$ controller, and the value of $\lambda_{2}$ sets their position in the frequency axis. The smaller the value of $x$, the longer the distance between the zero and pole. These two values $\left(x, \lambda_{2}\right)$ depend on the value of $\mu$ (see Eq. (23)). For a fixed pair $\left(x, \lambda_{2}\right)$, the higher the absolute value of $\mu$, the higher the slope of the magnitude of the $P D^{\mu}$ controller and the higher the maximum phase that the compensator can give. After some simple calculations, the expressions for $x$ and $\lambda_{2}$ can be given by

$x=\frac{a-1}{a(a-1)+b^{2}}, \quad \lambda_{2}=\frac{a(a-1)+b^{2}}{b \omega_{c}}$.

Studying the conditions for $a$ and $b$ to find a solution, it can be concluded that a lead compensator is obtained when $a>1$ and $b>0$, and a lag compensator when $\left(1-\sqrt{1-4 b^{2}}\right) / 2<a<\left(1+\sqrt{1-4 b^{2}}\right) / 2$ and $-1 / 2<b$ $<0$. Fig. 11 shows these lead and lag regions in the complex plane for the integer order compensator $C^{\prime}\left(\mathrm{j} \omega_{c g}\right)(\mu=1)$.

Let us focus on the lead compensation. It is clear that for the conventional lead compensator $(\mu=1)$ the vector $a+$ $j b=a_{1}+j b_{1}$ is perfectly known through the knowledge of the plant $G_{f l a t}\left(\mathrm{j} \omega_{c g}\right)$ (relay test) and the specifications of phase margin and gain crossover frequency required for the

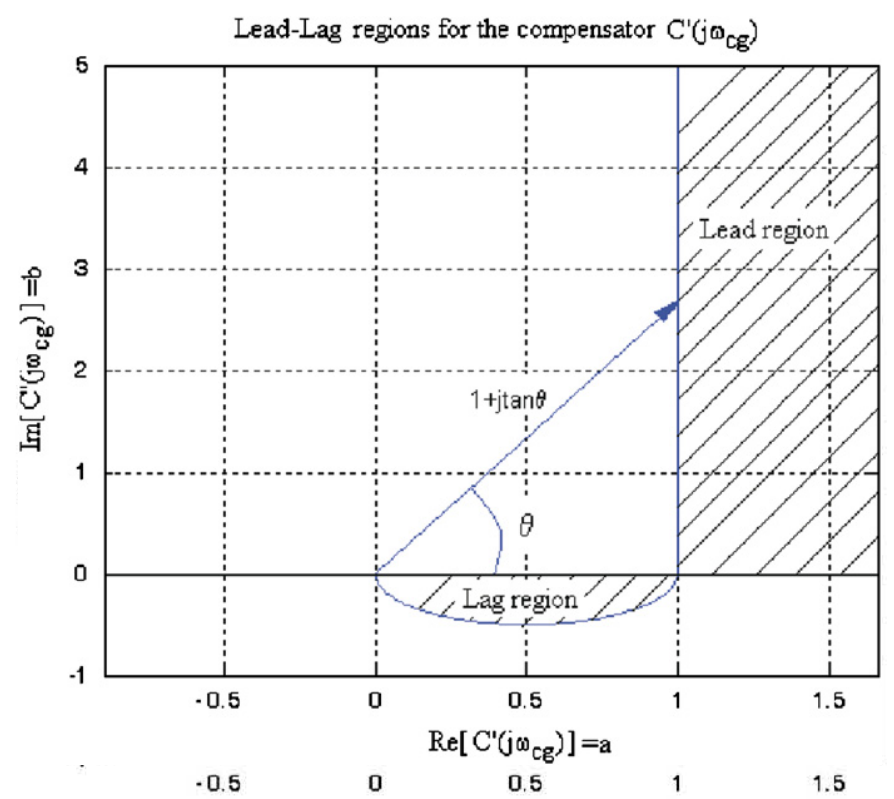

Fig. 11. Lead and lag regions for $\mathrm{C}_{\mathrm{fth}}^{\mathrm{the}}$ integer order compensator $C^{\prime}\left(\mathrm{j} \omega_{c g}\right)(\mu=1)$. system, as it can be seen in (23). Knowing the pair $(a, b)$, the values of $x$ and $\lambda_{2}$ are directly obtained by (24), and the compensator design is finished.

As shown in Fig. 11, the vector $1+j \tan \theta$ defines the borderline of the lead region. Using the polar form of this vector

$\sqrt{1+\tan ^{2} \theta} \mathrm{e}^{\mathrm{j} \theta}=\frac{1}{\cos \theta} \mathrm{e}^{\mathrm{j} \theta}$,

and expressing the vector $\left(a_{1}+\mathrm{j} b_{1}\right)^{1 / \mu}$ in its polar form

$\left(\sqrt{a_{1}^{2}+b_{1}^{2}}\right)^{1 / \mu} \mathrm{e}^{\mathrm{j}\left(\tan ^{-1}\left(b_{1} / a_{1}\right) / \mu\right)}=\rho^{1 / \mu} \mathrm{e}^{\mathrm{j}(\delta / \mu)}$,

where $\rho=\left(\sqrt{a_{1}^{2}+b_{1}^{2}}\right)$ and $\delta=\tan ^{-1}\left(b_{1} / a_{1}\right)$, the following relationships can be established from (23), making (25) equal to (26)

$\delta=\theta \mu$,

$\rho^{1 / \mu}=\frac{1}{\cos \theta} \Rightarrow 1=\rho\left[\cos \left(\frac{\delta}{\mu}\right)\right]^{\mu}$.

Then, solving numerically the function $1=\rho[\cos (\delta / \mu)]^{\mu}$, the lead compensation regions in the complex plane for different positive values of $\mu$ are obtained, as shown in Fig. 12. The procedure followed to obtain the curves is the one described next. For each value of $\mu$ (a specific curve) the pairs $\left(a_{1}, b_{1}\right)$ that form the curve are obtained. Since $\rho=\left(\sqrt{a_{1}^{2}+b_{1}^{2}}\right)$ and $\delta=\tan ^{-1}\left(b_{1} / a_{1}\right)$, it is clear that $a_{1}=$ $\rho \cos (\delta)$ and $b_{1}=\rho \sin (\delta)$. Besides, $\delta$ and $\rho$ are functions of $\mu$, that is, $\delta=\theta \mu$ and $\rho=(1 / \cos (\delta / \mu))^{\mu}$. Now, a vector $\theta$ is defined with increasing values in the range $0<\theta<\pi / 2$. Remember that $\theta$ defines the borderline of the integer lead region and, therefore, $\pi / 2$ is its maximum limit. For each value of $\theta$ a pair $(\delta, \rho)$ is obtained, and then a point $\left(a_{1}, b_{1}\right)$ is defined. Repeating these steps for all the values of $\theta$, all the points $\left(a_{1}, b_{1}\right)$ forming the curve will be obtained. After

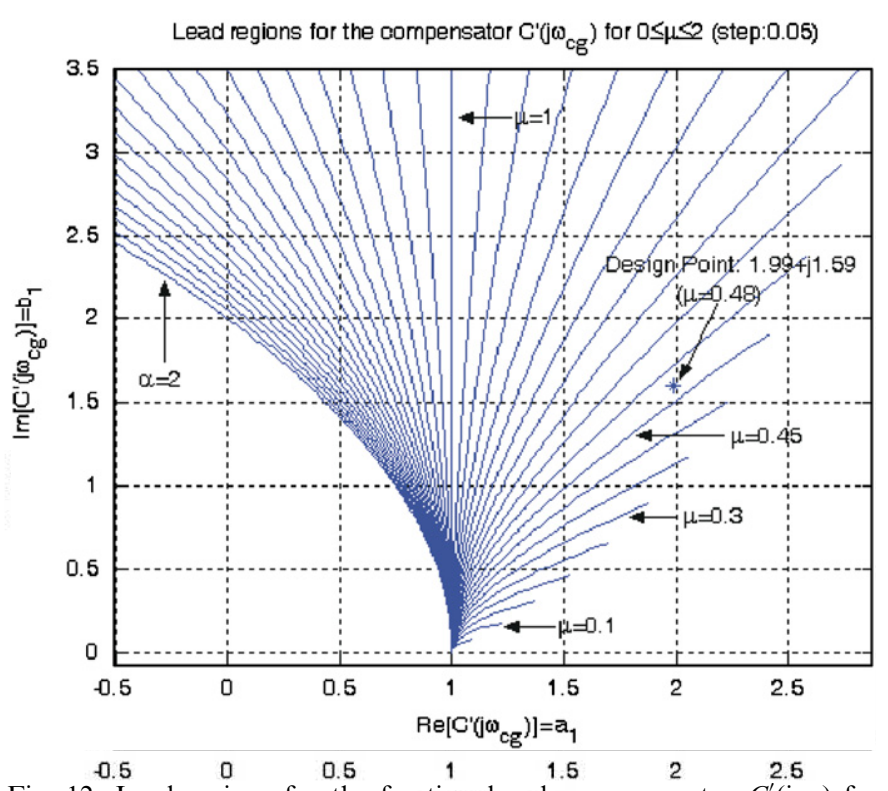

Fig. 12. Lead regions for the fractipnal order compensator $C^{\prime}\left(\mathrm{j} \omega_{c}\right)$ for $0 \leqslant \mu \leqslant 2$. 
that, a new value of $\mu$ is selected and the process is repeated to obtain a new curve.

The zone to the right of each curve is the lead region, and any design point in this zone can be fulfilled with a fractional order compensator having a value of $\mu$ equal or bigger than the one defining the curve which passes through the design point $\left(\mu_{\min }\right)$. For instance, for the design point in Fig. 12, the value of $\mu_{\text {min }}$ is 0.48 . By choosing the minimum value $\mu_{\min }$, the distance between the zero and the pole of the compensator will be the maximum possible (minimum value of parameter $x$, a positive value very close to zero). In this case, the phase curve of the compensator is the flattest possible and variations in a frequency range centered at $\omega_{c g}$ will not produce a significant phase change, improving the robustness of the open-loop system regarding its iso-damping property. Let us remember that the phase of $G_{f l a t}(s)$ around $\omega_{c g}$ was already flat due to the effect of the controller $P I^{\lambda}(s)$ and, therefore, it is the shape of the phase curve of the fractional order lead compensator $\left(P D^{\mu}\right)$ that affects the robustness of the system to gain variations.

Let us then sum up how the $P I^{\lambda} D^{\mu}$ controller is autotuned. The following steps can be solved by a simple computer, using a data acquisition system to control and monitor the real process (as explained in the section for experimental results in this paper). A PLC could also be used for the determination of the parameters of the controller, due to the simplicity of the equations involved in the auto-tuning method.

1. Once the specifications of design are given $\left(\omega_{c g}\right.$ and $\left.\varphi_{m}\right)$, the relay test is applied to the plant and the resulting pairs $\left(\theta_{n}, \omega_{n}\right)$ obtained from the $n$ iterations of the test are saved and used for the calculation of the phase and magnitude of the plant at each frequency $\omega_{n}$ (following Eqs. (16) and (17)). As explained previously, these values are used for the obtaining of the slope of the plant phase $v(18)$. With the value of the slope, the parameters $\lambda$ and $\lambda_{1}$ of the $P I^{\lambda}$ controller are directly obtained by Eqs. (20) and (22). Then, the system $G_{\text {flat }}\left(\mathrm{j} \omega_{c g}\right)$ is obtained.

2. Once the system $G_{f l a t}\left(\mathrm{j} \omega_{c g}\right)$ is defined, and according to Eq. (23), the parameters of the fractional order compensator in (15) are obtained by simple calculations summarized next, following the robustness feature explained in this section.

3. Select a very small initial value of $\mu$, for example, $\mu=$ 0.05 . For this initial value, calculate the value of $x$ and $\lambda_{2}$ using the relations in (23) and (24).

4. If the value of $x$ obtained is negative, then the value of $\mu$ is increased a fixed step and step 2 is repeated again. The smaller the fixed increase of $\mu$ the more accurate the selection of the parameter $\mu_{\min }$. Repeat step 2 until the value of $x$ obtained is positive.

5. Once a positive value of $x$ is obtained, the value of $\mu$ must be recorded as $\mu_{\min }$. This value of $x$ will be close to zero and will ensure the maximum flatness of the phase curve of the compensator (iso-damping constraint). The value of $\lambda_{2}$ corresponding to this value $\mu_{\min }$ is also recorded.

Therefore, all the parameters of the $P I^{\lambda} D^{\mu}$ controller have been obtained through this iterative process. Then, the controller is implemented and starts to control the process through the switch illustrated in Fig. 9, concluding the auto-tuning procedure.

\subsection{Formulation of the resulting $P I^{t \lambda} D^{\mu}$ controller}

Once the parameters of the fractional order $P I^{\lambda} D^{\mu}$ controller of Eq. (13) are obtained by following the design methods explained above, these parameters can be identified with those ones of the standard $P I^{\lambda} D^{\mu}$ controller given by

$C_{s t d}(s)=k_{p}\left(1+\frac{1}{T_{i} s}\right)^{\lambda}\left(1+\frac{T_{d} s}{1+s T_{d} / N}\right)^{\mu}$.

Carrying out some calculations in (28), the following transfer function is obtained:

$C_{s t d}(s)=\frac{k_{p}}{\left(T_{i}\right)^{\lambda}}\left(\frac{T_{i} s+1}{s}\right)^{\lambda}\left(\frac{T_{d}(1+1 / N) s+1}{\left(T_{d} / N\right) s+1}\right)^{\mu}$.

Comparing expressions (13) and (28), the relations obtained are $T_{i}=\lambda_{1}, k_{p}=k^{\prime} /\left(\lambda_{1}\right)^{\lambda}, N=(1-x) / x$ and $T_{d}=\lambda_{2}(1-x)$.

In the next section the auto-tuning method proposed here is illustrated by experimental examples of application.

\subsection{Experimental results by using the auto-tuning method}

For the implementation of the auto-tuning method proposed the following devices have been used, showing a connection scheme in Fig. 13:

- Data acquisition board AD 512, by Humusoft, running on Matlab 5.3 and using the real time toolbox "RealTime Windows Target'.

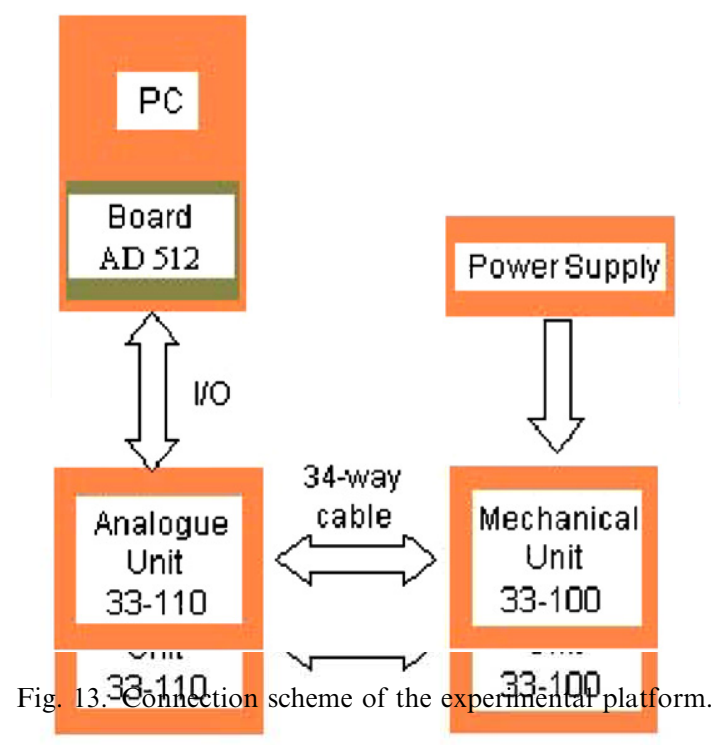


- A computer Pentium II, $350 \mathrm{MHz}, 64 \mathrm{M}$ RAM, which supports the data acquisition board and where the programs run for the implementation of the method proposed.

- A servomotor 33-002 by Feedbak, in Fig. 14, that consists of: (a) a mechanical unit 33-100, which constitutes the servo, strictly speaking, (b) an analogue unit 33-110, which connects to the mechanical unit through a 34-way ribbon cable which carries all power supplies and signals enabling the normal circuit interconnections to be made on the analogue unit and (c) a power supply $01-100$ for the system. The mechanical unit has a brake whose position changes the gain of the system, that is, the break acts like a load to the motor. This break will be used to test the robustness of the controlled system to gain variations.

Specifications of gain crossover frequency, phase margin and robustness to plant gain variations are given. In this case, the desired gain crossover frequency is $\omega_{c g}=2.3 \mathrm{rad} / \mathrm{s}$. The relay has an output amplitude of $d=6$, without hysteresis, $\varepsilon=0$. The two initial values $\left(\theta_{-1}\right.$ and $\theta_{0}$ ) of the delay used to reach the frequency specified are 0.1 and $0.04 \mathrm{~s}$, respectively. After several iterations the output signal shown in Fig. 15 is obtained.

The value of the delay $\theta_{a}$ obtained for the selection of the frequency specified is $\theta_{a}=0.2326 \mathrm{~s}$, and the corresponding frequency is $\omega_{u}=2.2789 \mathrm{rad} / \mathrm{s}$. The amplitude and period of this oscillatory signal are $a=1.8701$ and $T_{u}=2.7571 \mathrm{~s}$, respectively. Therefore, the magnitude and phase of the plant estimated through the relay experiment at the frequency $\omega_{u}=2.2789 \mathrm{rad} / \mathrm{s}$ are $\left|G\left(\mathrm{j} \omega_{u}\right)\right|_{\mathrm{dB}}=$ $-12.2239 \mathrm{~dB}$ and $\arg \left(G\left(\mathrm{j} \omega_{u}\right)\right)=-149.6328^{\circ}$, respectively.
Measuring experimentally the frequency response of the system in order to validate these values, a magnitude of $-11.8556 \mathrm{~dB}$ and a phase of $-150.2001^{\circ}$ are obtained. So, only a slight error is committed in the estimation. Next, a fractional order $P I^{\lambda} D^{\mu}$ controller is designed with the proposed tuning method to obtain a phase margin $\varphi_{m}=$ $60^{\circ}$ at the gain crossover frequency $\omega_{u}=2.2789 \mathrm{rad} / \mathrm{s}$. The gain of the controller will be fixed to 1 , that is, $k^{\prime}=$ $k_{c} x^{\alpha}=1$.

The first step is the design of the fractional order $P I^{\lambda}$ part, in Eq. (14). For that purpose the slope of the phase of the plant, $v$, is estimated by using the expression (18). The slope obtained in this case is $v=-0.2568 \mathrm{rad} /(\mathrm{rad} / \mathrm{s})$. With the value of the slope and applying the criterion described for the fractional order $P I^{\lambda}$ controller (see Eqs. (20) and (22)), the controller that cancels the slope of the phase curve of the plant is

$P I^{\lambda}(s)=\left(\frac{0.4348 s+1}{s}\right)^{0.8468}$

At the frequency $\omega_{u}$ this fractional order $P I^{\lambda}$ controller has a magnitude of $-3.5429 \mathrm{~dB}$, a phase of $-38.3291^{\circ}$ and a phase slope of 0.2568 . Therefore, the estimated system $G_{\text {flat }}(s)$ has a magnitude of $-15.7668 \mathrm{~dB}$ and a phase of $-187.9619^{\circ}$. These values can be easily obtained through the values of the magnitude and phase of the plant estimated by the relay test at the frequency $\omega_{u}$ and the magnitude and phase of the controller $P I^{\lambda}(s)$ at the same frequency. Next, the controller $P D^{\mu}(s)$ is designed to fulfill the specifications of phase margin and gain crossover frequency required for the controlled system. Following the iterative process described previously, the resulting

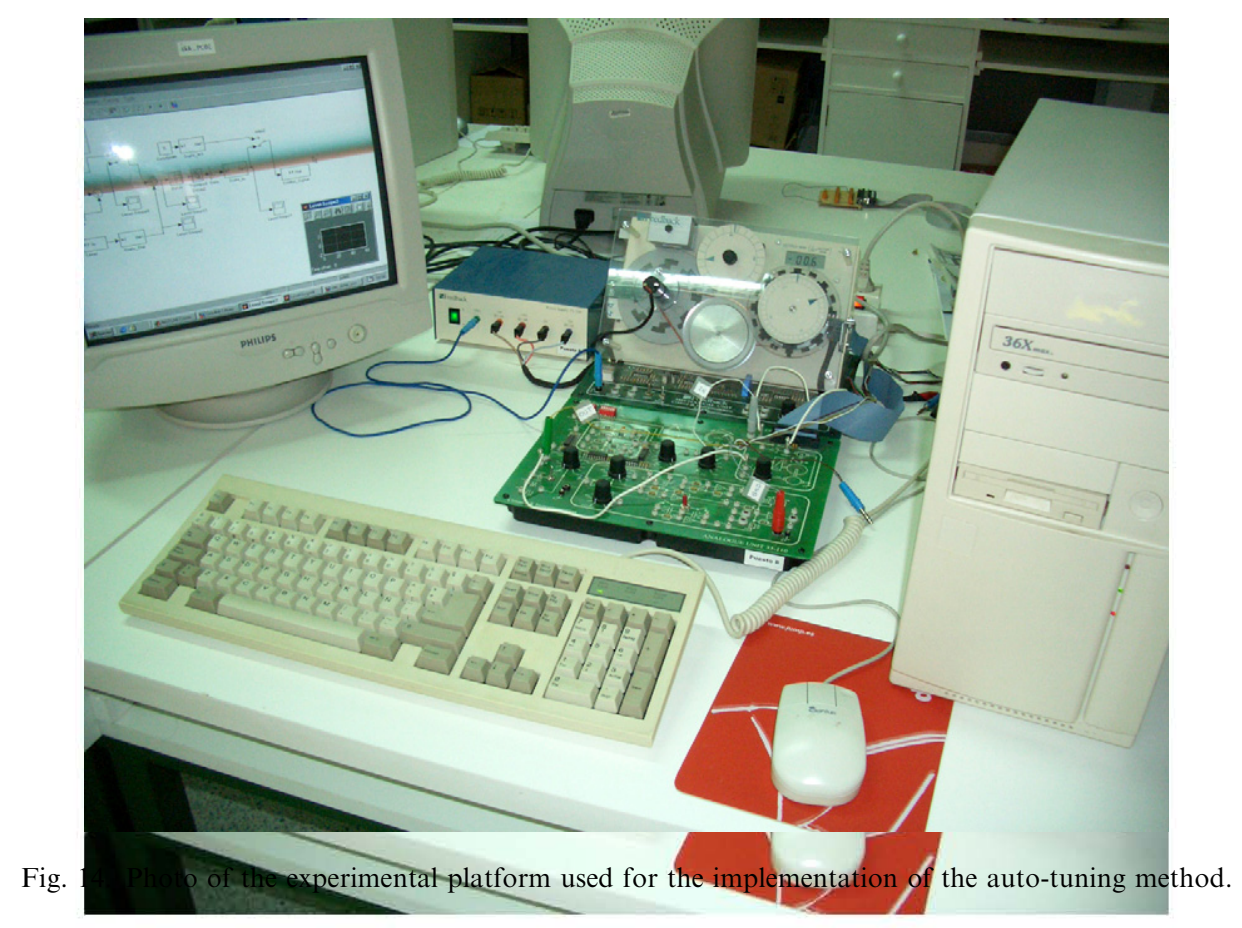



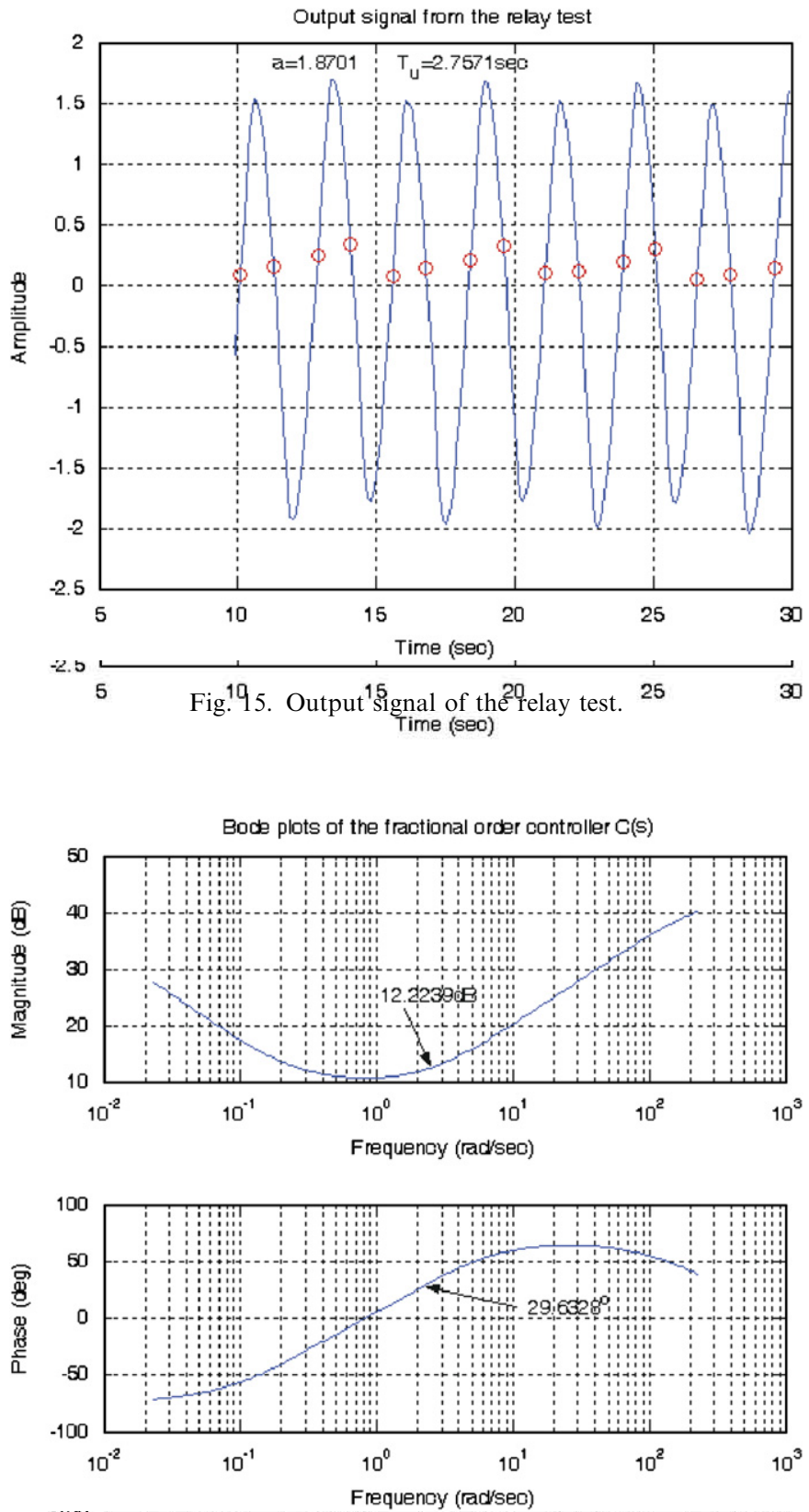

Fig. 16. Bode plots of the ${ }^{10^{0}}$ fractional order controller $^{10^{-1}} C(s) . \quad 10^{3}$

controller is given by

$P D^{\mu}(s)=\left(\frac{4.0350 s+1}{0.0039 s+1}\right)^{0.8160}$.

At the frequency $\omega_{u}=2.2789 \mathrm{rad} / \mathrm{s}$ the controller $P D^{\mu}(s)$ has a magnitude of $15.7668 \mathrm{~dB}$ and a phase of $67.9619^{\circ}$.

Then, the resulting total controller $C(s)$ is the following one

$C(s)=\left(\frac{0.4348 s+1}{s}\right)^{0.8468}\left(\frac{4.0350 s+1}{0.0039 s+1}\right)^{0.8160}$.

The Bode plots of $C(s)$ are shown in Fig. 16. The magnitude and phase of this controller at the frequency $\omega_{u}$

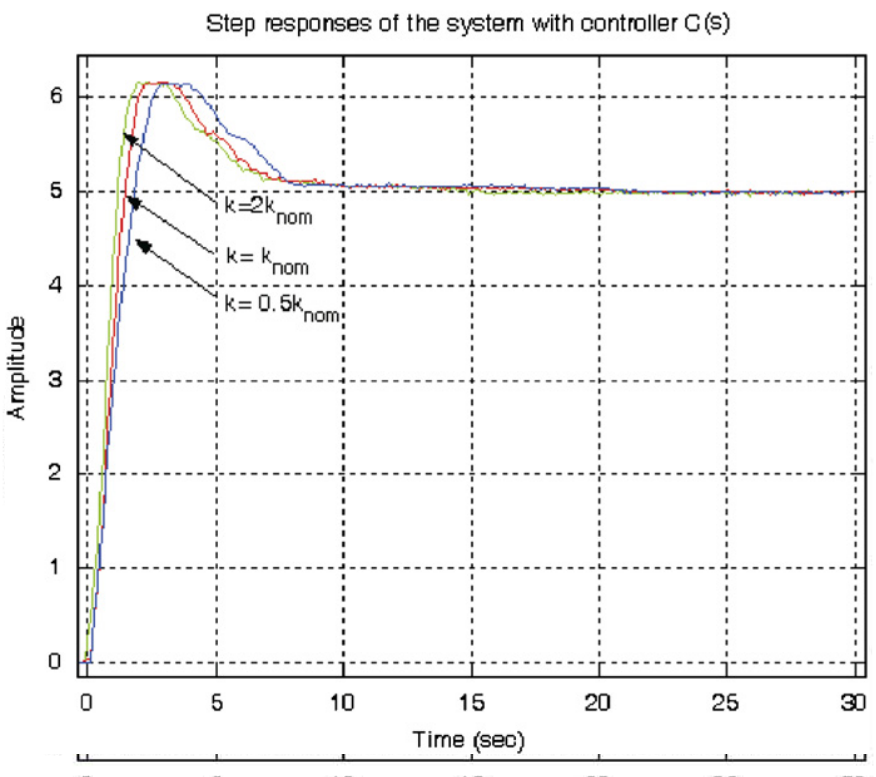

qig. 17. Step responses of the 15 system with controller ${ }^{25} C(s)$.

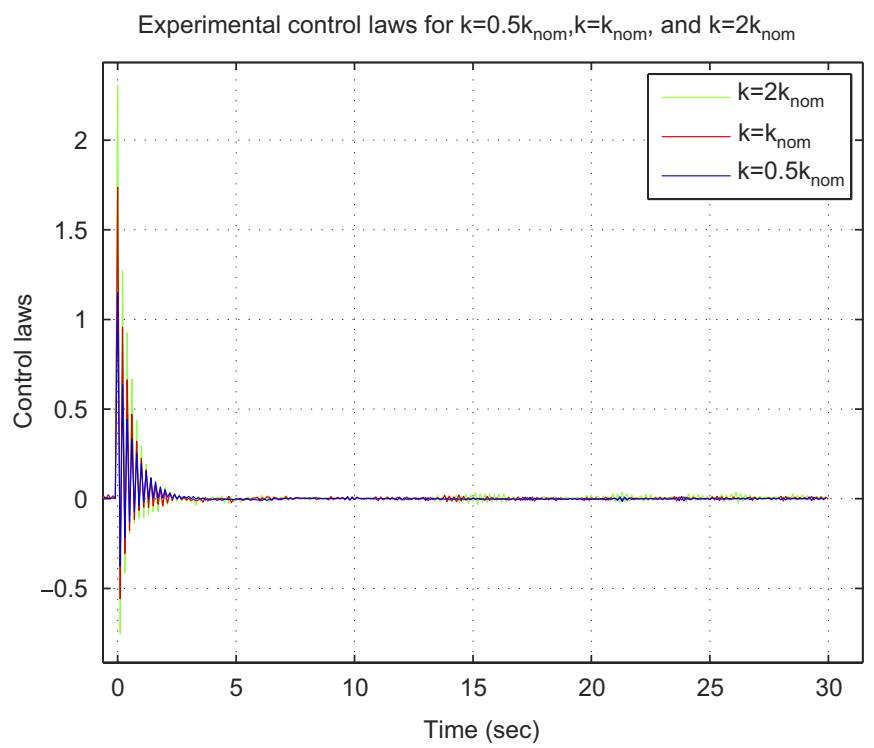

Fig. 18. Experimental control laws of the controlled system with controller $C(s)$.

are $12.2239 \mathrm{~dB}$ and $29.6328^{\circ}$, respectively. Therefore, the open-loop system $F(s)$ has a phase margin of $60^{\circ}$ and a magnitude of $0 \mathrm{~dB}$ at the gain crossover frequency $\omega_{u}=2.2789 \mathrm{rad} / \mathrm{s}$, fulfilling the design specifications.

For the implementation of the resulting fractional order controller $C(s)$, the frequency domain identification technique using Matlab function invfreqs is applied again. An integer-order transfer function is obtained which fits the frequency response of the fractional order controller in the range $\omega \in\left(10^{-2}, 10^{2}\right)$, with 3 poles/zeros for the $P I^{\lambda}$ part and 3 poles/zeros for the $P D^{\mu}$ part. Later, the discretization of this continuous approximation is made by using the Tustin rule with prewarping, with a sampling time $T_{s}=$ $0.01 \mathrm{~s}$ and prewarp frequency $\omega_{c g}$. With this controller the 


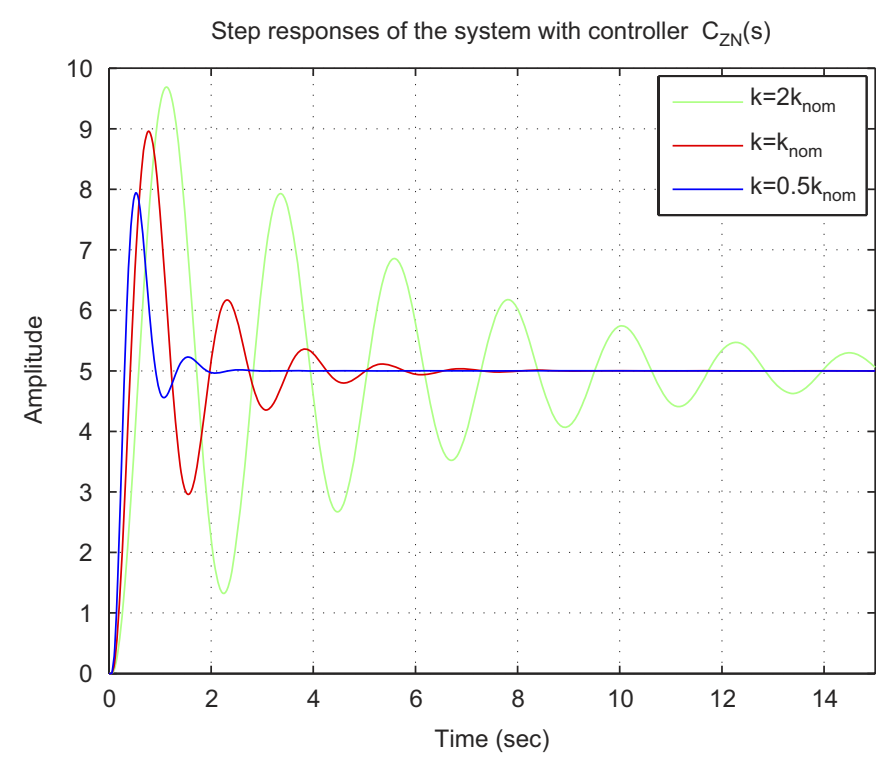

Fig. 19. Step responses of the system with controller $C_{Z N}(s)$.

phase of the open-loop system $F(s)$ is the flattest possible, ensuring the maximum robustness to variations in the gain of the plant, as can be seen in the step responses of the controlled system for $k=k_{\text {nom }}$ (nominal gain), $k=2 k_{\text {nom }}$ and $k=0.5 k_{\text {nom }}$ (Fig. 17). The gain variations are provoked by changing the position of the motor brake. Fig. 18 shows the control laws of the system for the different gains. It can be observed that for this gain range this control strategy is very suitable, since the peak of the control laws is much lower than $10 \mathrm{~V}$, the saturation voltage of the motor. Comparing the step responses with the ones obtained (in simulation) with the PID controller $C_{Z N}(s)=22.1010\left(1+\frac{1}{0.55 s}+0.1375 s\right)$ designed by the second method of Ziegler-Nichols (Fig. 19), the better performance of the system with the fractional order controller $C(s)$ can be observed.

\section{Conclusions}

First of all, a synthesis method for fractional order $P I^{\lambda} D^{\mu}$ controllers has been developed to fulfill five different design specifications for the closed-loop system, that is, two more specifications than in the case of a conventional PID controller. An optimization method to tune the controller has been used for that purpose, based on a nonlinear function minimization subject to some given nonlinear constraints. Experimental results show that the requirements are totally fulfilled for the platform to be controlled. Thus, advantage has been taken of the fractional orders $\lambda$ and $\mu$ to fulfill additional specifications of design, ensuring a robust performance of the controlled system to gain changes and noise.

Besides, an auto-tuning method for the fractional order $P I^{\lambda} D^{\mu}$ controller using the relay test has been proposed. This method allows a flexible and direct selection of the parameters of the controller through the knowledge of the magnitude and phase of the plant at the frequency of interest, obtained with the relay test. Specifications of gain crossover frequency, $\omega_{c g}$, and phase margin, $\varphi_{m}$, can be fulfilled with a robustness property based on the flatness of the phase curve of the open-loop system, guaranteeing the iso-damping property of the time response of the system to gain variations. Again, the experimental results illustrate the effectiveness of this method.

\section{Acknowledgment}

This work has been financially supported by the Spanish Research Project 2PR02A024 of the Junta de Extremadura (Spain).

\section{References}

Axtell, M., \& Bise, E. (1990). Fractional calculus applications in control systems. In IEEE 1990 national aerospace and electronics conference, New York, USA (pp. 563-566).

Barbosa, R., Tenreiro, J., \& Ferreira, I. (2004a). PID controller tuning using fractional calculus concepts. Fractional Calculus and Applied Analysis, 7(2), 119-134.

Barbosa, R., Tenreiro, J. A., \& Ferreira, I. M. (2004b). Tuning of PID controllers based on Bode's ideal transfer function. Nonlinear Dynamics, 38, 305-321.

Barbosa, R. S., Tenreiro, J. A., \& Ferreira, I. M. (2003). A fractional calculus perspective of PID tuning. In ASME 2003 design engineering technical conferences and computers and information in engineering conference, Chicago, USA.

Bode, H. (1940). Relations between attenuation and phase in feedback amplifier design. Bell System Technical Journal, 19, 421-454.

Bode, H. W. (1945). Network analysis and feedback amplifier design. Princeton, NJ: Van Nostrand.

Calderón, A. J. (2003). Fractional control of a power electronic buck converter, Ph.D. thesis, Escuela de Ingenierías Industriales, Universidad de Extremadura, Badajoz, Spain.

Calderón, A. J., Vinagre, B. M., \& Feliu, V. (2003). Linear fractional order control of a DC-DC buck converter. In ECC 2003: European control conference 2003, Cambridge, UK.

Caponetto, R., Fortuna, L., \& Porto, D. (2002). Parameter tuning of a non-integer order PID controller. In 15th international symposium on mathematical theory of networks and systems, Notre Dame, Indiana.

Caponetto, R., Fortuna, L., \& Porto, D. (2004). A new tuning strategy for a non-integer order PID controller. In First IFAC workshop on fractional differentiation and its application (pp. 168-173). Bordeaux, France: ENSEIRB.

Chen, Y. Q. (2006). Ubiquituos fractional order controls. In The second IFAC symposium on fractional derivatives and applications (IFAC FDA06), Porto, Portugal.

Chen, Y. Q., \& Moore, K. L. (2002). Discretization schemes for fractional order differentiators and integrators. IEEE Transactions on Circuits and Systems I: Fundamental Theory and Applications, 49(3), 363-367.

Chen, Y. Q., \& Moore, K. L. (2005). Relay feedback tuning of robust PID controllers with iso-damping property. IEEE Transactions on Systems, Man, and Cybernetics, Part B, 35(1), 23-31.

Chen, Y. Q., Moore, K. L., Vinagre, B. M., \& Podlubny, I. (2004). Robust PID controller autotuning with a phase shaper. In First IFAC workshop on fractional differentiation and its applications (pp. 162-167). Bordeaux, France: ENSEIRB.

Chen, Y. Q., Vinagre, B. M., \& Monje, C. A. (2003). A proposition for the implementation of non-integer PI controllers. In Thematic action 
"systems with non-integer derivations". Bordeaux, France: LAPENSEIRB.

Chen, Y. Q., Vinagre, B. M., \& Podlubny, I. (2004). Continued fraction expansion approaches to discretizing fractional order derivatives-An expository review. Nonlinear Dynamics, 38(1-4), 155-170.

Feliu, V., Rivas, R., \& Sánchez, L. (2007). Fractional robust control of main irrigation canals with variable dynamic parameters. Control Engineering Practice, 15(6), 673-686.

Fonseca, N. M., \& Tenreiro, J. A. (2003). Fractional-order hybrid control of robotic manipulators. In 11th international conference on advanced robotics, Coimbra, Portugal (pp. 393-398).

Franklin, G., Powell, J., \& Naeini, A. (1986). Feedback control of dynamic systems. Reading, MA: Addison-Wesley.

Hang, C. C., Åström, K. J., \& Wang, Q. G. (2002). Relay feedback autotuning of process controllers-A tutorial review. Journal of Process Control, 12, 143-162.

Lanusse, P., Poinot, T., Cois, O., Oustaloup, A., \& Trigeassou, J. (2003). Tuning of an active suspension system using a fractional controller and a closed-loop tuning. In 11th international conference on advanced robotics, Coimbra, Portugal (pp. 258-263).

Laroche, E., \& Knittel, D. (2005). An improved linear fractional model for robustness analysis of a winding system. Control Engineering Practice, 13(5), 659-666.

Leu, J. F., Tsay, S. Y., \& Hwang, C. (2002). Design of optimal fractionalorder PID controllers. Journal of the Chinese Institute of Chemical Engineers, 33(2), 193-202.

Levine, W. S. (1996). The control handbook. Boca Raton, New York: CRC Press, IEEE Press.

Malti, R., Aoun, M., Cois, O., Oustaloup, A., \& Levron, F. (2003). $H_{2}$ norm of fractional differential systems. In ASME 2003 design engineering technical conferences and computers and information in engineering conference, Chicago, USA.

Manabe, S. (1961). The non-integer integral and its application to control systems. ETJ of Japan, 6(3-4), 83-87.

MathWorks. (2000a). Matlab optimization toolbox, version 2.1. User's guide. The MathWorks, Inc.

MathWorks. (2000b). Matlab signal processing toolbox. User's guide. The MathWorks, Inc.

Monje, C. A. (2006). Design methods of fractional order controllers for industrial applications, Ph.D. thesis, Escuela de Ingenierías Industriales, Universidad de Extremadura, Badajoz, Spain.

Monje, C. A., Calderón, A. J., \& Vinagre, B. M. (2002). PI vs fractional DI control: First results. In CONTROLO 2002: 5th Portuguese conference on automatic control, Aveiro, Portugal (pp. 359-364).

Monje, C. A., Calderón, A. J., Vinagre, B. M., Chen, Y. Q., \& Feliu, V. (2004). On fractional $P I^{\lambda}$ controllers: Some tuning rules for robustness to plant uncertainties. Nonlinear Dynamics, 38(1-4), 369-381.

Monje, C. A., Vinagre, B. M., Calderón, A. J., Feliu, V., \& Chen, Y. Q. (2005). Auto-tuning of fractional lead-lag compensators. In 16th IFAC world congress, Prague.

Oustaloup, A. (1991). CRONE control: Robust control of non-integer order. Paris: Hermes.

Oustaloup, A. (1995). Non-integer derivation. Paris: Hermes.

Oustaloup, A., Levron, F., Nanot, F., \& Mathieu, B. (January 2000). Frequency-band complex noninteger differentiator: Characterization and synthesis. IEEE Transactions on Circuits and Systems I: Fundamental Theory and Applications, 47 (1), 25-40.

Oustaloup, A., Mathieu, B., \& Lanusse, P. (1995). The CRONE control of resonant plants: Application to a flexible transmission. European Journal of Control, 1(2), 113-121.
Petráš, I., \& Hypiusova, M. (2002). Design of fractional-order controllers via $H_{\infty}$ norm minimisation. Selected Topics in Modelling and Control, $3,50-54$.

Petráš, I., \& Vinagre, B. M. (2002). Practical application of digital fractional-order controller to temperature control. Acta Montanistica Slovaca, 7(2), 131-137.

Petráš, I., Vinagre, B. M., Dorčák, L., \& Feliu, V. (2002). Fractional digital control of a heat solid: Experimental results. In International Carpathian control conference, Malenovice, Czech Republic (pp. 365-370).

Pintér, J. (1996). Global optimization in action. Dordrecht, The Netherlands: Kluwer Academic Publishers.

Podlubny, I. (1999a). Fractional differential equations. San Diego: Academic Press

Podlubny, I. (1999b). Fractional-order systems and $P I^{\lambda} D^{\mu}$ controllers. IEEE Transactions on Automatic Control, 44(1), 208-214.

Podlubny, I., Petráš, I., Vinagre, B. M., O'Leary, P., \& Dorčák, L. (2002). Analogue realizations of fractional-order controllers. Nonlinear Dynamics, 29(1-4), 281-296.

Pommier, V., Musset, R., Lanusse, P., \& Oustaloup, A. (2003). Study of two robust control for an hydraulic actuator. In ECC 2003: European control conference 2003, Cambridge, UK.

Sabatier, J., \& Oustaloup, A. (2003). Implementation of a thermal platform for the test of non-integer systems. In Conference on education of technologies and information and systems sciences (pp. 361-364). Toulouse, France: Univerité Paul Sabatier.

Tan, K. K., Huang, S., \& Ferdous, R. (2002). Robust self-tuning PID controller for nonlinear systems. Journal of Process Control, 12(7).

Tenreiro, J. A., \& Azenha, A. (1998). Fractional-order hybrid control of robot manipulators. In 1998 IEEE international conference on systems, man and cybernetics: Intelligent systems for humans in a cyberworld, San Diego, California, USA (pp. 788-793).

Valério, D. (2001). Fractional order robust control: An application. In Student forum, Porto (pp. 25-28).

Valério, D. (2005). Fractional robust system control, Ph.D. thesis, Instituto Superior Técnico, Universidade Técnica de Lisboa.

Vinagre, B., Podlubny, I., Dorčák, L., \& Feliu, V. (2000). On fractional PID controllers: A frequency domain approach. In IFAC workshop on digital control. Past, present and future of PID control, Terrasa, Spain (pp. 53-58).

Vinagre, B., Podlubny, I., Hernández, A., \& Feliu, V. (2000). Some approximations of fractional order operators used in control theory and applications. Fractional Calculus and Applied Analysis, 3(3), 231-248.

Vinagre, B. M. (2001). Modelling and control of dynamic systems characterized by integro-differential equations of fractional order, Ph.D. thesis, UNED, Madrid, Spain.

Vinagre, B. M., Monje, C. A., \& Calderón, A. J. (2002). Fractional order systems and fractional order control actions. In 41 st conference on decision and control. Tutorial workshop 2: Fractional calculus applications in automatic control and robotics, Las Vegas.

Vinagre, B. M., Monje, C. A., Calderón, A. J., Chen, Y. Q., \& Feliu, V. (2004). The fractional integrator as a reference function. In First IFAC workshop on fractional differentiation and its applications (pp. 150-155) Bordeaux, France: ENSEIRB.

Vinagre, B. M., Petráš, I., Merchán, P., \& Dorčák, L. (2001). Two digital realizations of fractional controllers: Application to temperature control of a solid. In ECC 2001: European control conference 2001, Porto, Portugal (pp. 1764-1767).

Yago Sánchez, B. S. (1999). Fractional-PID control for active reduction of vertical tail buffeting. Technical Report, Saint Louis University. 